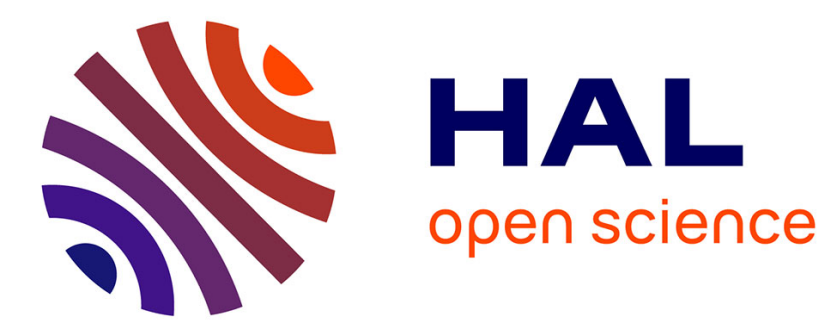

\title{
In-line flow-induced vibrations of a rotating cylinder
}

\author{
Rémi Bourguet, David Lo Jacono
}

\section{To cite this version:}

Rémi Bourguet, David Lo Jacono. In-line flow-induced vibrations of a rotating cylinder. Journal of Fluid Mechanics, 2015, 781, pp. 127-165. 10.1017/jfm.2015.477 . hal-01310722

\section{HAL Id: hal-01310722 \\ https://hal.science/hal-01310722}

Submitted on 3 May 2016

HAL is a multi-disciplinary open access archive for the deposit and dissemination of scientific research documents, whether they are published or not. The documents may come from teaching and research institutions in France or abroad, or from public or private research centers.
L'archive ouverte pluridisciplinaire HAL, est destinée au dépôt et à la diffusion de documents scientifiques de niveau recherche, publiés ou non, émanant des établissements d'enseignement et de recherche français ou étrangers, des laboratoires publics ou privés. 


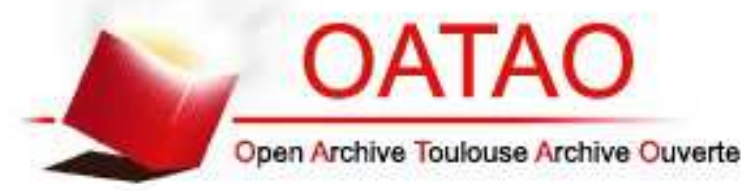

\section{Open Archive TOULOUSE Archive Ouverte (OATAO)}

OATAO is an open access repository that collects the work of Toulouse researchers and makes it freely available over the web where possible.

This is an author-deposited version published in : http://oatao.univ-toulouse.fr/ Eprints ID : 15752

To link to this article : DOI:10.1017/jfm.2015.477

URL : http://dx.doi.org/10.1017/jfm.2015.477

\section{To cite this version :}

Bourguet, Rémi and Lo Jacono, David In-line flow-induced vibrations of a rotating cylinder. (2015) Journal of Fluid Mechanics, vol. 781. pp. 127-165. ISSN 0022-1120

Any correspondence concerning this service should be sent to the repository administrator: staff-oatao@ listes-diff.inp-toulouse.fr 


\title{
In-line flow-induced vibrations of a rotating cylinder
}

\author{
Rémi Bourguet ${ }^{1} \uparrow$ and David Lo Jacono ${ }^{1}$ \\ ${ }^{1}$ Institut de Mécanique des Fluides de Toulouse, CNRS, UPS and Université de Toulouse, \\ Toulouse, 31400, France
}

The flow-induced vibrations of an elastically mounted circular cylinder, free to oscillate in the direction parallel to the current and subjected to a forced rotation about its axis, are investigated by means of two- and three-dimensional numerical simulations, at a Reynolds number equal to 100 based on the cylinder diameter and inflow velocity. The cylinder is found to oscillate up to a rotation rate (ratio between the cylinder surface and inflow velocities) close to 2 (first vibration region), then the body and the flow are steady until a rotation rate close to 2.7 where a second vibration region begins. Each vibration region is characterized by a specific regime of response. In the first region, the vibration amplitude follows a bell-shaped evolution as a function of the reduced velocity (inverse of the oscillator natural frequency). The maximum vibration amplitudes, even though considerably augmented by the rotation relative to the non-rotating body case, remain lower than 0.1 cylinder diameters. Due to their trends as functions of the reduced velocity and to the fact that they develop under a condition of wake-body synchronization or lock-in, the responses of the rotating cylinder in this region are comparable to the vortex-induced vibrations previously described in the absence of rotation. The symmetry breaking due to the rotation is shown to directly impact the structure displacement and fluid force frequency contents. In the second region, the vibration amplitude tends to increase unboundedly with the reduced velocity. It may become very large, higher than 2.5 diameters in the parameter space under study. Such structural oscillations resemble the galloping responses reported for non-axisymmetric bodies. They are accompanied by a dramatic amplification of the fluid forces compared to the non-vibrating cylinder case. It is shown that body oscillation and flow unsteadiness remain synchronized and that a variety of wake topologies may be encountered in this vibration region. The low-frequency, large-amplitude responses are associated with novel asymmetric multi-vortex patterns, combining a pair and a triplet or a quartet of vortices per cycle. The flow is found to undergo three-dimensional transition in the second vibration region, with a limited influence on the system behaviour. It appears that the transition occurs for a substantially lower rotation rate than for a rigidly mounted cylinder.

Key words: flow-structure interactions, vortex streets, wakes 


\section{Introduction}

Flexible or flexibly mounted bodies with bluff cross-section and immersed in a cross-current exhibit flow-induced vibrations (FIV). Such phenomena are omnipresent in nature (e.g. oscillations of plants in wind or water streams) and are also encountered in a myriad of civil, wind, offshore and nuclear engineering applications, for instance, the vibrations of mooring lines and cables exposed to ocean currents. The increased mean drag forces, the fatigue damage and possible failure of the structures due to these vibrations have motivated a number of studies aiming at clarifying the fundamental mechanisms of FIV, as collected in Blevins (1990), Naudascher \& Rockwell (1994) and Païdoussis, Price \& de Langre (2010). The present work focuses on the FIV of a flexibly mounted rigid circular cylinder subjected to a forced rotation. This system may be regarded as a paradigm of symmetry breaking in fluid-structure interaction, as further explicated in the following.

The unsteady fluid forces exerted on a bluff body due to vortex shedding in its wake can result in structural oscillations. This type of FIV, referred to as vortex-induced vibrations (VIV), has been extensively investigated through the canonical problem of a non-rotating rigid circular cylinder forced or free to oscillate within a cross-flow (Bishop \& Hassan 1964; King, Prosser \& Johns 1973; Griffin \& Ramberg 1976; Bearman 1984, 2011; Naudascher 1987; Ongoren \& Rockwell 1988; Williamson \& Roshko 1988; Mittal \& Tezduyar 1992; Hover, Techet \& Triantafyllou 1998; Carberry, Sheridan \& Rockwell 2001; Cetiner \& Rockwell 2001; Jeon \& Gharib 2001; Okajima, Kosugi \& Nakamura 2002; Sarpkaya 2004; Williamson \& Govardhan 2004; Klamo, Leonard \& Roshko 2006; Lucor \& Triantafyllou 2008; Dahl et al. 2010; Leontini, Lo Jacono \& Thompson 2011; Cagney \& Balabani 2013; Navrose \& Mittal 2013; Konstantinidis 2014). A non-rotating circular cylinder is not susceptible to movement-induced vibrations (MIV), which allows VIV to be examined independently of this other type of FIV associated with aerodynamically unstable configurations (e.g. Païdoussis et al. 2010). VIV of non-axisymmetric bodies potentially exposed to MIV, for instance, a cylinder with a square or rectangular cross-section, have also been analysed in several studies (Naudascher 1987; Obasaju, Ermshaus \& Naudascher 1990; Okajima et al. 1999; Nemes et al. 2012; Zhao, Cheng \& Zhou 2013). VIV naturally appear both in the direction normal to the current (cross-flow direction) and in the direction parallel to the current (in-line direction). They persist when the body is free to move only in one direction. The typical amplitudes of these vibrations are of the order of one body diameter in the cross-flow direction and one or more orders of magnitude lower in the in-line direction. For a flexibly mounted rigid body, VIV usually appear over a well-defined range of the reduced velocity, i.e. inverse of the oscillator natural frequency non-dimensionalized by the inflow velocity and the body diameter. In this range, vortex formation and body oscillation are synchronized: cross-flow VIV generally occur at the vortex shedding frequency while in-line VIV may develop at this frequency or twice this frequency depending on the symmetry of the system, which determines the frequency ratio between the vortex shedding and in-line fluid force fluctuation. The condition of wake-body synchronization is referred to as lock-in. Under lock-in, the vortex formation frequency can depart from the shedding frequency observed downstream of the stationary body (Strouhal frequency) and the wake pattern can be considerably altered. The vibration frequency can also shift away from the natural frequency (e.g. Williamson \& Govardhan 2004).

Contrary to VIV, MIV do not involve a mechanism of lock-in between the body oscillation and vortex formation. They are due to an aerodynamic instability where the motion of the body tends to enhance the energy transfer from the flow to the structure, 
leading to self-excited oscillations (Naudascher \& Rockwell 1994; Païdoussis et al. 2010). The resulting low-frequency vibrations increase in amplitude unboundedly with the reduced velocity and are generally referred to as galloping responses (Den Hartog 1932; Parkinson \& Smith 1964; Mukhopadhyay \& Dugundji 1976; Nakamura \& Tomonari 1977). Such structural responses may occur in the in-line and cross-flow directions (e.g. Abdel-Rohman 1992). Due to the absence of coupling with vortex shedding, galloping oscillations can often be predicted through quasi-steady theory (Parkinson \& Smith 1964). However, for specific ranges of the structure properties, VIV and galloping regions may sometimes overlap and novel vibration regimes may emerge (Bearman et al. 1987; Corless \& Parkinson 1988; Nemes et al. 2012; Mannini, Marra \& Bartoli 2014; Zhao et al. 2014a).

In the present study, the axial symmetry of the body geometry is preserved but the cross-flow symmetry of the fluid-structure system is broken by applying a forced rotation to the flexibly mounted circular cylinder. The impact of an imposed rotation on the wake patterns and fluid forcing has been well documented in the case of a rigidly mounted circular cylinder (Coutanceau \& Ménard 1985; Badr et al. 1990; Chew, Cheng \& Luo 1995; Kang, Choi \& Lee 1999; Stojković, Breuer \& Durst 2002; Mittal \& Kumar 2003; Pralits, Brandt \& Giannetti 2010). Different flow regimes have been identified depending on the rotation rate $\alpha$, defined as the ratio between the cylinder surface velocity and the oncoming flow velocity. In particular, previous works have shown that the alternating vortex shedding associated with the von Kármán instability vanishes above a rotation rate $\alpha \approx 2$. This phenomenon is accompanied by the suppression of the periodic fluctuations of the fluid forces. The flow three-dimensional transition scenario is dramatically modified by the forced rotation (Mittal 2004; El Akoury et al. 2008; Meena et al. 2011). Compared to the stationary cylinder case, a variety of new steady and unsteady three-dimensional wake patterns has been evidenced in the rotation rate - Reynolds number $(R e)$ parameter space (Radi et al. 2013; Rao et al. 2013b, 2015). It was shown that the rotation may cause three-dimensional transition down to $\operatorname{Re} \approx 30$, versus $\operatorname{Re} \approx 190$ in the absence of actuation (Pralits, Giannetti \& Brandt 2013; Rao et al. 2013a). In the above mentioned studies and in the following, the Reynolds number is based on the cylinder diameter and on the oncoming flow velocity.

The case of an elastically mounted circular cylinder, forced to rotate and free to oscillate both in the in-line and cross-flow directions, has been the object of a few studies. Zhao, Cheng \& Lu (2014b) examined numerically the influence of the imposed rotation on the VIV regime for $\alpha \leqslant 1$ and $R e=150$. They reported a substantial alteration of the flow and structural responses. More specifically, they noted a significant increase of the maximum amplitudes of vibration, especially in the in-line direction, and a switch of the in-line response frequency which is equal to the vortex shedding frequency once the cylinder rotates, instead of twice the shedding frequency for $\alpha=0$. In this range of low rotation rates, the in-line oscillation amplitude remains however lower than one cylinder diameter and the vibrations of the two degrees-of-freedom oscillator are comparable to the VIV developing in the non-rotating case (lock-in condition, bell-shaped evolution of the response). Through experiments and numerical simulations, Stansby \& Rainey (2001) studied the impact of higher rotation rates on the responses of the two degrees-of-freedom oscillator, in the Reynolds number range 200-4700. For $\alpha \in[2,5]$, the cylinder was shown to exhibit large oscillations, with peak amplitudes sometimes higher than 10 diameters in each direction. Free vibrations thus appear beyond the critical value of $\alpha$ associated with vortex shedding suppression in the rigidly mounted cylinder case. Yogeswaran 
\& Mittal (2011) observed similar responses for $\alpha=4.5$ and $R e=200$, on the basis of numerical simulation results. These vibrations, characterized by amplitudes growing continuously with reduced velocity and low frequencies, are comparable to the galloping responses encountered for non-axisymmetric bodies, such as cylinders with rectangular cross-sections. A large number of vortices may form during each vibration cycle. These previous works indicate that responses analogous to VIV and galloping oscillations may develop for the two degrees-of-freedom oscillator, depending on the rotation rate. No systematic analyses of flow patterns and forcing have been reported in the above studies for $\alpha>1$.

A simplification of the physical configuration was considered in a previous numerical study by focusing on a single degree-of-freedom oscillator (Bourguet \& Lo Jacono 2014). In this prior work, a detailed investigation the system behaviour was performed, in the rotation rate-reduced velocity parameter space, for an elastically mounted rotating cylinder allowed to move in the cross-flow direction only, at $R e=100$. The cylinder exhibits free cross-flow oscillations from $\alpha=0$ up to a rotation rate close to 4 . In contrast to the observations made in the two degrees-of-freedom configuration, the forced rotation was shown to have a very limited impact on the single degree-of-freedom oscillator response in the low $\alpha$ range $(\alpha \leqslant 1)$, as recently corroborated by Zhao et al. (2014b) at $R e=150$. At higher values of $\alpha$, it was found that the rotation induces an amplification of the cross-flow vibration which may reach 1.9 diameters, i.e. three times the maximum amplitude in the non-rotating case. Over the entire range of $\alpha$, the vibration amplitude presents a bell-shaped trend as a function of the reduced velocity and the free vibrations occur under a condition of wake-body synchronization similar to the lock-in condition driving non-rotating cylinder VIV. As a result, contrary to the two degrees-of-freedom configuration, no galloping-like response was observed when the body was restrained to move in the cross-flow direction. Novel asymmetric flow patterns were identified in the wake of the rotating cylinder subjected to free cross-flow vibrations. It was also shown that the flow tends to remain two-dimensional under transverse oscillation in a range of $\alpha$ where the rotation causes three-dimensional transition when the body does not vibrate.

The present work aims at extending the previous analysis concerning the single degree-of-freedom oscillator (Bourguet \& Lo Jacono 2014) by considering the case where the flexibly mounted rotating cylinder is allowed to move in the in-line direction, instead of the cross-flow direction. The possible appearance of galloping-like oscillations in such configuration is under question. More generally, the impact of the forced rotation on the circular cylinder in-line VIV and the alteration of the interaction mechanisms previously described in the absence of actuation remain to be explored. Prior works concerning non-rotating cylinders have emphasized that an in-line oscillation may lead to a range of wake dynamics including periodic, quasiperiodic and chaotic states (Perdikaris, Kaiktsis \& Triantafyllou 2009; Leontini et al. 2011; Tudball-Smith et al. 2012; Leontini, Lo Jacono \& Thompson 2013). The wake patterns occurring under the combination of forced rotation and free in-line vibration still need to be investigated. Another element to clarify in this context is the flow three-dimensional transition and its potential influence on the structural responses. To address these aspects, the behaviour of the coupled flow-structure system is examined by means of two- and three-dimensional numerical simulations, over a wide range of rotation rates and reduced velocities. The Reynolds number is chosen equal to 100 in reference to the previously mentioned work concerning the cross-flow vibrations of a rotating cylinder. 


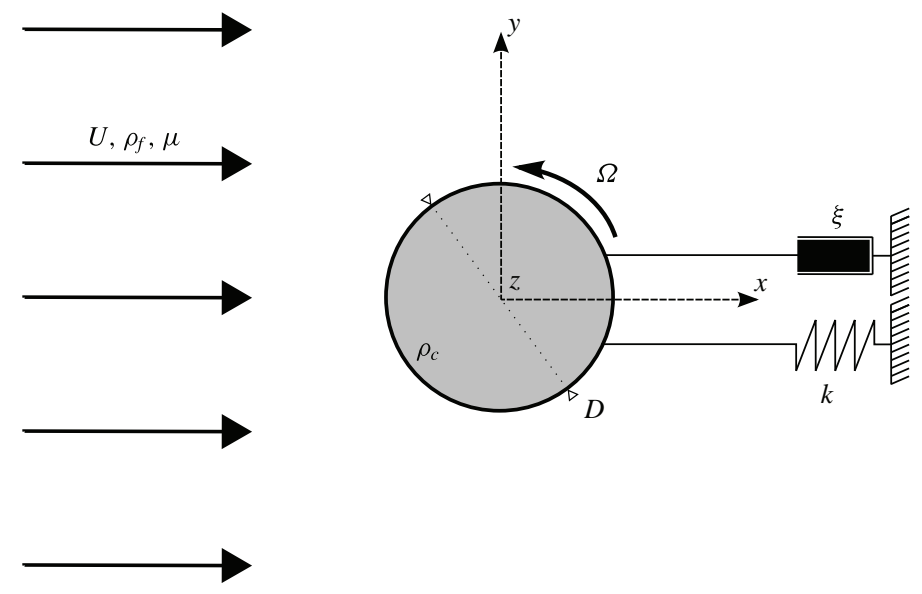

FIGURE 1. Sketch of the physical configuration.

The paper is organized as follows. The physical model and the numerical method are presented in $\S 2$. The cylinder responses are quantified in $\S 3$. The flow downstream of the flexibly mounted rotating cylinder is studied in $\S 4$. The fluid forces are analysed in $\S 5$. The main findings of this work are summarized in $\S 6$.

\section{Formulation and numerical method}

The physical configuration and its modelling are described in $\S 2.1$. The numerical method employed and its validation are presented in $\S 2.2$.

\subsection{Fluid-structure system}

The physical system is similar to that considered in a previous work concerning the flow-induced vibrations of a rotating cylinder (Bourguet \& Lo Jacono 2014) except that in the present study, the body is free to oscillate in the in-line direction instead of the cross-flow direction. A sketch of the configuration is presented in figure 1. The elastically mounted rigid cylinder has a circular cross-section. It is immersed in a cross-flow which is parallel to the $x$ axis and normal to the cylinder axis ( $z$ axis). The flow is assumed to be incompressible. The Reynolds number based on the oncoming flow velocity $(U)$ and cylinder diameter $(D), R e=\rho_{f} U D / \mu$, where $\rho_{f}$ and $\mu$ denote the fluid density and viscosity, respectively, is equal to 100 , as in the above mentioned study. At $R e=100$, the flow remains two-dimensional over a wide region of the parameter space investigated. The two-dimensional Navier-Stokes equations are employed over the entire parameter space to predict the flow dynamics. In order to study the flow three-dimensional transition at high rotation rates, and its potential influence on the system behaviour, the three-dimensional Navier-Stokes equations are also considered in selected points of the parameter space. In the three-dimensional case, an aspect ratio of $L / D=10$, where $L$ is the cylinder length in the spanwise direction ( $z$ axis), is selected as a reasonable balance between the wavelength of the flow three-dimensional pattern (of the order of $1-2 D$ ) and the numerical cost.

The cylinder is fixed in the cross-flow direction ( $y$ axis) and free to oscillate in the in-line direction ( $x$ axis). The structural stiffness, damping ratio and mass per unit length are designated by $k, \xi$ and $\rho_{c}$, respectively. In the following, all the physical 
variables are non-dimensionalized by the cylinder diameter, the oncoming flow velocity and the fluid density. The non-dimensional mass of the structure is defined as $m=\rho_{c} / \rho_{f} D^{2}$. The actual ratio between the mass of the cylinder and the mass of the displaced fluid is equal to $4 m / \pi$. The non-dimensional cylinder displacement, velocity and acceleration are denoted by $\zeta, \dot{\zeta}$ and $\ddot{\zeta}$, respectively. The in-line and cross-flow force coefficients are defined as $C_{x}=2\left\langle F_{x}\right\rangle / \rho_{f} D U^{2}$ and $C_{y}=2\left\langle F_{y}\right\rangle / \rho_{f} D U^{2}$, where $F_{x}$ and $F_{y}$ are the in-line and cross-flow dimensional sectional fluid forces, and \langle\rangle the span-averaging operator. $\left\langle F_{x}\right\rangle$ and $\left\langle F_{y}\right\rangle$ are thus equal to the integrals of the pressure and viscous stress terms over the cylinder surface, normalized by its length. In the two-dimensional case, $\left\langle F_{x}\right\rangle=F_{x}$ and $\left\langle F_{y}\right\rangle=F_{y}$. The body dynamics is governed by a forced second-order oscillator equation which can be expressed as follows, in non-dimensional formulation:

$$
\ddot{\zeta}+\frac{4 \pi \xi}{U^{\star}} \dot{\zeta}+\left(\frac{2 \pi}{U^{\star}}\right)^{2} \zeta=\frac{C_{x}}{2 m} .
$$

$U^{\star}$ denotes the reduced velocity, $U^{\star}=1 / f_{n}$, where $f_{n}$ is the non-dimensional natural frequency in vacuum, $f_{n}=D / 2 \pi U \sqrt{k / \rho_{c}}$. As in the prior study concerning cross-flow vibrations, the structural damping is set equal to zero $(\xi=0)$ to allow maximum amplitude oscillations and $m$ is set equal to 10 .

The cylinder is subjected to a forced, counter-clockwise, steady rotation about its axis. The imposed rotation is controlled by the rotation rate $\alpha=\Omega D / 2 U$, where $\Omega$ is the angular velocity of the cylinder.

The behaviour of the system is explored in the $\left(\alpha, U^{\star}\right)$ parameter space. The following set of rotation rates is investigated, $\alpha \in\{0,0.5,1,1.5,2,2.5,2.625,2.7$, $2.75,3,3.25,3.5\}$. This set includes the unsteady/steady flow transition for a rigidly mounted rotating cylinder $(\alpha \approx 1.8$ at $R e=100$ (Kang et al. 1999; Stojković et al. 2003)) but excludes the three-dimensional transition in this case, which was found to occur for $\alpha \approx 3.7$ at $R e=100$ (Pralits et al. 2013; Rao et al. 2013a; Bourguet \& Lo Jacono 2014). The flow three-dimensional transition may however appear at lower rotation rates when the body oscillates in the in-line direction, as shown in the present work. The reduced velocity ranges from 1 to 23 .

\subsection{Numerical simulation method}

The numerical method employed is the same as in the prior study concerning cross-flow vibrations (Bourguet \& Lo Jacono 2014). Descriptions of the simulation approach, boundary conditions and discretizations, as well as a detailed validation were reported in this previous study. Only a brief summary of the method and some additional validation results are presented here. The coupled flow-structure equations are solved by the parallelized code Nektar, which is based on the spectral/hp element method (Karniadakis \& Sherwin 1999). A large rectangular computational domain is considered ( $350 \mathrm{D}$ downstream and $250 \mathrm{D}$ in front, above and below the cylinder) in order to avoid any spurious blockage effects due to domain size. A no-slip condition is applied at the cylinder surface. Flow periodicity conditions are employed on the side (spanwise) boundaries in the three-dimensional case.

The evolution of the time-averaged force coefficients as a function of the spectral element polynomial order, for the cases of both a rigidly mounted cylinder for $\alpha=3.5$ (the largest rotation rate considered in this paper) and of a flexibly mounted cylinder for $\left(\alpha, U^{\star}\right)=(3.5,23)$ (i.e. in the region of maximum vibration amplitude, as shown 

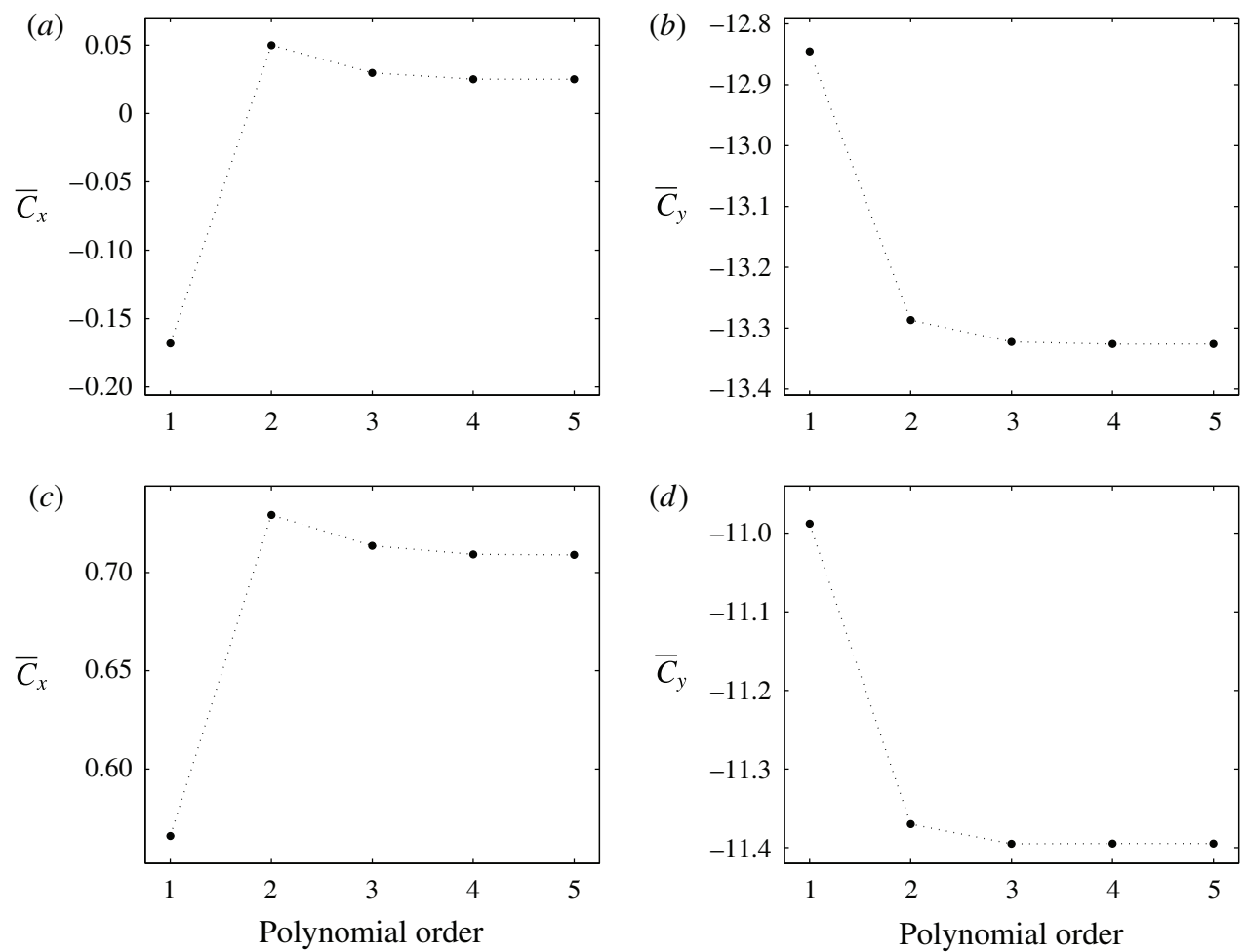

FIgURE 2. Time-averaged $(a, c)$ in-line and $(b, d)$ cross-flow force coefficients as functions of the polynomial order, in the cases of $(a, b)$ a rigidly mounted rotating cylinder for $\alpha=3.5$ and $(c, d)$ a flexibly mounted cylinder for $\left(\alpha, U^{\star}\right)=(3.5,23)$, at $R e=100$.

in $\S 3$ ), are plotted in figure 2. The imposed rotation cancels the vortex shedding in the former case and the flow is steady, thus $\bar{C}_{x}=C_{x}$ and $\bar{C}_{y}=C_{y}$, where ${ }^{-}$denotes time-averaged values. These plots show that an increase from order 4 to 5 has no significant influence on the results. Hence, a polynomial order equal to 4 is selected. For the three-dimensional simulations, 64 complex Fourier modes are employed in the spanwise direction. Additional three-dimensional simulations in the area of the parameter space where the flow is found to be three-dimensional show that doubling the spanwise resolution (i.e. 128 Fourier modes) has only a negligible impact on the results. For instance, less than $0.1 \%$ of relative difference is noted for the vibration amplitude in the region of maximum vibration amplitude $\left(\left(\alpha, U^{\star}\right)=(3.5,23)\right)$.

All the simulations are initialized with the established periodic flow past a stationary cylinder at $R e=100$. Then the forced rotation is started and the body is released. The analysis is based on time series of more than 40 oscillation cycles, collected after convergence of the time-averaged and root mean square (RMS) values of the fluid force coefficients and body displacement.

\section{Structural responses}

A description of the body responses is reported in this section. Focus is placed first on the time-averaged displacement of the cylinder $(\$ 3.1)$ and then on its oscillatory motion $(\S 3.2)$. 

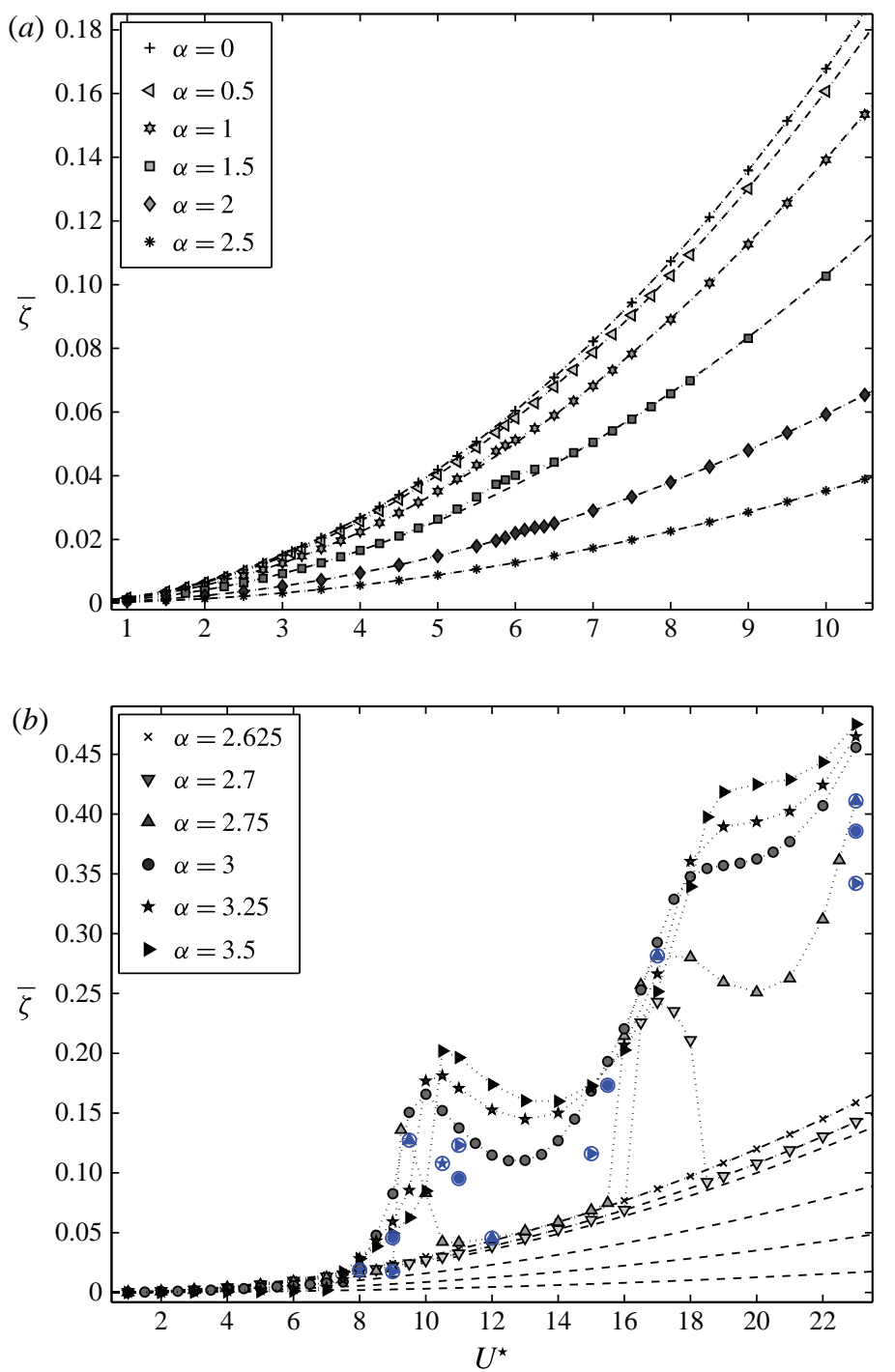

FIgURE 3. (Colour online) Time-averaged displacement as a function of the reduced velocity for $(a) \alpha \leqslant 2.5$ and $(b) \alpha>2.5$. For each rotation rate, a dashed line indicates the displacement associated with the time-averaged in-line force in the rigidly mounted cylinder case. The three-dimensional simulation results are denoted by circled blue symbols.

\subsection{Time-averaged displacement}

The time-averaged displacement of the body is plotted in figure 3 as a function of the reduced velocity for each rotation rate. For more clarity in the presentation, the results for $\alpha \leqslant 2.5$ and $\alpha>2.5$ are plotted separately. In this figure and in the following, the two-dimensional simulation results are represented by uncircled greyscale symbols. The three-dimensional simulation results are denoted by the same marker shapes as the two-dimensional ones, but the symbols are circled and coloured in blue. By considering the time-averaged form of the structure dynamics (2.1), the 
time-averaged displacement of the cylinder can be expressed as $\bar{\zeta}=U^{\star 2} \bar{C}_{x} / 8 \pi^{2} m$. An estimate of $\bar{\zeta}$, based on this expression and the value of $\bar{C}_{x}$ in the rigidly mounted cylinder case, is indicated by a dashed line for each rotation rate in figure 3 . Due to the monotonic decrease of $\bar{C}_{x}$ as $\alpha$ increases (in the present range) in the rigidly mounted cylinder configuration (Stojković et al. 2003; Bourguet \& Lo Jacono 2014), the estimated time-averaged displacement tends to decrease when $\alpha$ increases. In spite of some minor deviations, as for instance around $U^{\star}=6$ for $\alpha=1.5$, the actual values of $\bar{\zeta}$ remain close to the estimates for $\alpha \leqslant 2.625$. In contrast, substantial deviations appear for higher rotation rates, which denotes a significant alteration of the time-averaged in-line force in this range of $\alpha$ when the cylinder is free to oscillate. The fluid forces are examined in $\$ 5$. The two- and three-dimensional simulation results are superimposed for $\alpha=2.75$ whereas some differences can be noted for $\alpha \geqslant 3$. The general trends identified via the two- and three-dimensional simulations are comparable, however the observed differences suggest that the flow undergoes a three-dimensional transition in the range of high rotation rates. This point is clarified in the next section.

\subsection{Free oscillatory motion}

An overview of the maximum oscillation amplitude of the cylinder about its time-averaged position is presented in figure $4(a)$, in the rotation rate-reduced velocity domain. In this plot, and in the following, $\sim$ denotes the fluctuation about the timeaveraged value. A first observation of the oscillatory responses allows identification of low-amplitude vibrations with bell-shaped evolutions as functions of $U^{\star}$ in the lower range of $\alpha$ and, after a range of intermediate rotation rates where no oscillations occur, a regime of large vibrations in the higher range of $\alpha$. For a sufficiently high value of the rotation rate, the vibration amplitude tends to increase unboundedly with $U^{\star}$ and thus departs from the above mentioned bell-shaped behaviour. For a better visualization of the two vibration regions appearing in the $\left(\alpha, U^{\star}\right)$ parameter space, a global map indicating the areas where the body is found to oscillate is reported in figure $4(b)$. A number of additional two-dimensional simulations have been performed to locate the limits of the vibration regions. However, it should be mentioned that these limits remain approximate since they are determined under two-dimensional flow assumption. Furthermore, they may also be subjected to hysteresis phenomena (Khalak \& Williamson 1999; Prasanth \& Mittal 2008). This map of the vibration regions differs from the map reported in a previous work concerning the cross-flow responses of a rotating cylinder (figure $6 c$ in Bourguet \& Lo Jacono 2014) where the area characterized by oscillations larger than $0.05 D$ was indicated. In the present map, the vibration regions denote the areas of the parameter space where the cylinder exhibits oscillations of any amplitudes. The area of the first vibration region where the response amplitude is larger than $0.01 D$ is enclosed by a dashed-dotted line.

The oscillations of the cylinder are periodic in all studied cases. The spectral analysis of the structural response is based on the power spectral density (PSD) of the time series of $\zeta$. The following nomenclature is adopted to describe the frequency content of the response. All the frequencies mentioned hereafter are non-dimensional. The frequency associated with the largest peak of $\zeta$ PSD is referred to as the dominant vibration frequency and denoted by $f$. The fundamental vibration frequency (i.e. frequency of the first harmonic component) of $\zeta$ is denoted by $f_{x}$. As discussed in this section, the response of the rotating cylinder is generally dominated by its fundamental frequency component $\left(f=f_{x}\right)$. In some rare cases, however, the second 
(a)

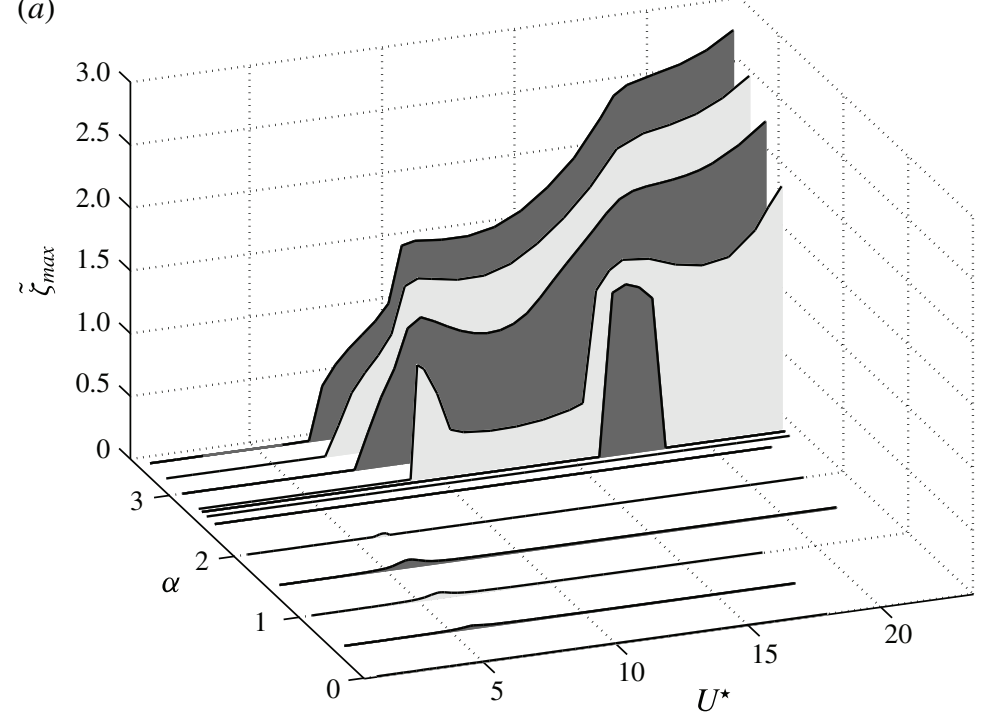

(b)

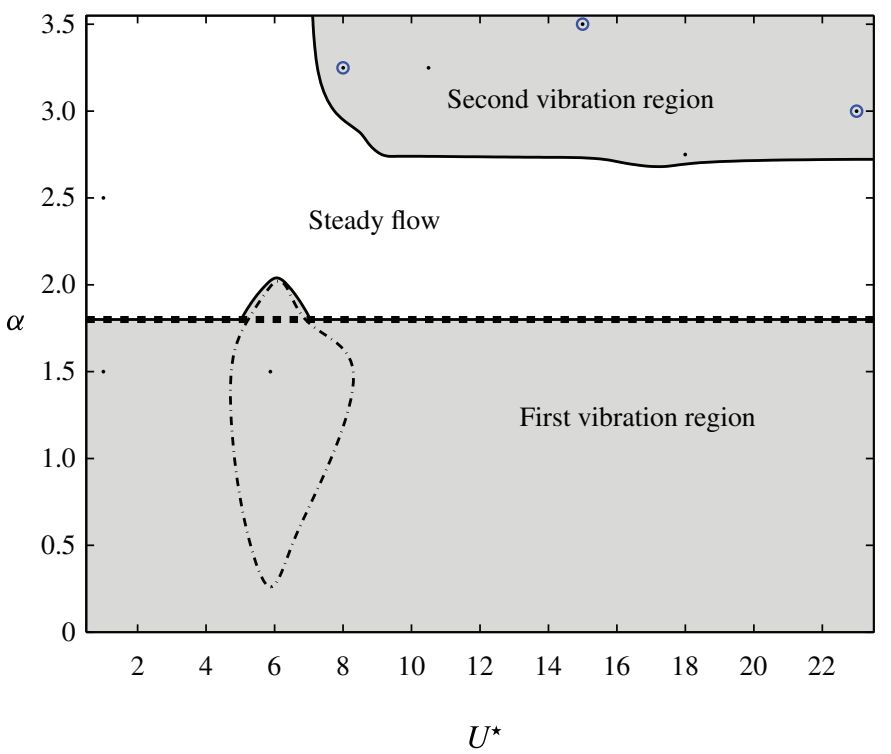

FIGURE 4. (Colour online) (a) Maximum amplitude of vibration and (b) vibration regions (grey colour), as functions of the rotation rate and reduced velocity. In $(b)$, a dashed-dotted line encloses the area of the first vibration region where the maximum vibration amplitude is larger than $0.01 D$; a dashed line represents the limit between the steady and unsteady flow regimes in the rigidly mounted cylinder case; black dots denote the cases visualized in figures 8-10 (two-dimensional results) while blue circles designate the cases presented in figures 13 and 14 (three-dimensional results).

harmonic component may dominate $\left(f=2 f_{x}\right)$. In the following sections, unless otherwise stated, the terms vibration frequency and response frequency refer to the dominant vibration frequency. It is recalled that $f_{n}$ designates the oscillator natural 

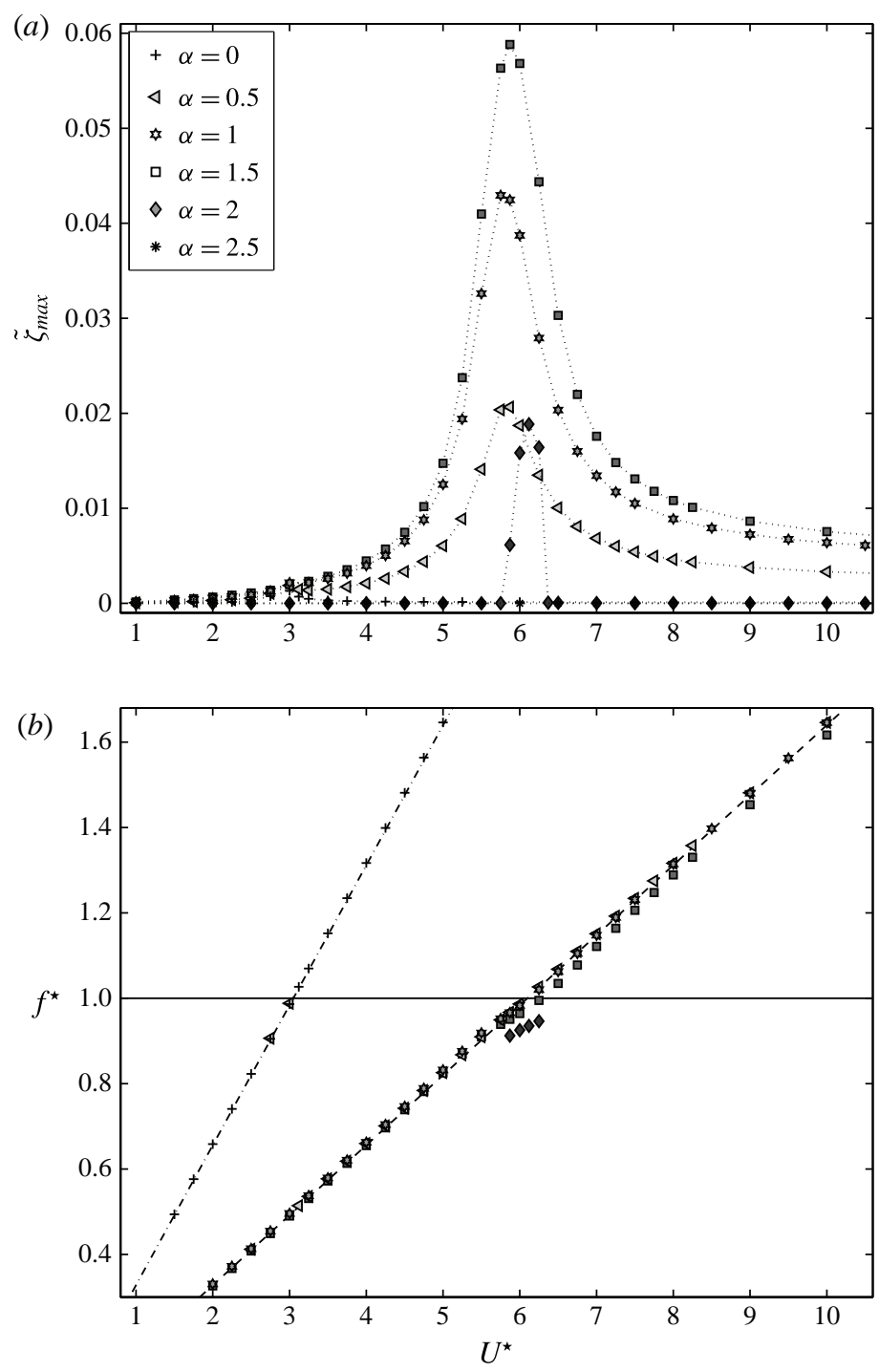

FIGURE 5. (a) Maximum amplitude of vibration and $(b)$ dominant vibration frequency normalized by the oscillator natural frequency, as functions of the reduced velocity, for $\alpha \leqslant 2.5$. In $(b)$, a dashed line indicates the frequency of vortex shedding in the stationary cylinder case (Strouhal frequency) normalized by the the oscillator natural frequency and a dashed-dotted line indicates twice this frequency ratio.

frequency. The deviation between the dominant vibration frequency and the natural frequency is quantified via the frequency ratio $f^{\star}=f / f_{n}$.

The maximum amplitude of vibration is plotted as a function of $U^{\star}$, for $\alpha \leqslant 2.5$ and $\alpha>2.5$ in figures $5(a)$ and $6(a)$, respectively. The associated value of $f^{\star}$ is reported in figures $5(b)$ and $6(b)$.

Below the critical rotation rate associated with the cancellation of vortex shedding in the rigidly mounted cylinder case $(\alpha \approx 1.8$ at $R e=100$, the dashed line in figure $4 b$ ), structural vibrations occur over the entire range of $U^{\star}$ investigated. The 

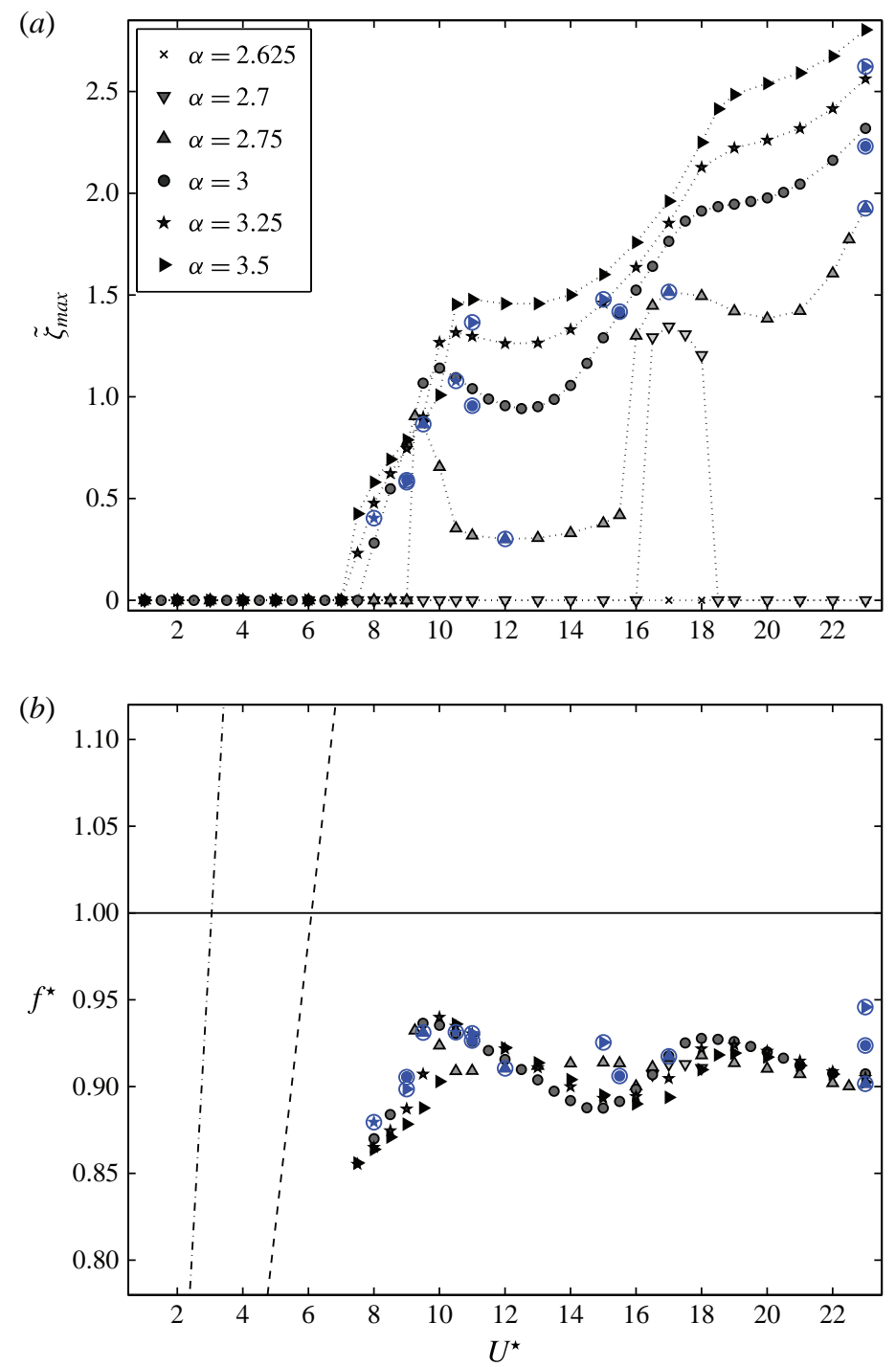

FIgURE 6. (Colour online) Same as figure 5, for $\alpha>2.5$. The three-dimensional simulation results are denoted by circled blue symbols.

oscillation amplitude remains however very small in this range of $\alpha(\tilde{\zeta}<0.01)$, except around the peaks visible in figure $4(a)$, in the region identified by a dashed-dotted line in figure $4(b)$. The persistence of some vibrations away from the interval of $U^{\star}$ associated with the peak amplitudes of response was reported for a rotating cylinder free to vibrate in the cross-flow direction or in both directions for $\alpha<1.8$ (Bourguet \& Lo Jacono 2014; Zhao et al. 2014b) and it is generally observed for non-rotating cylinders (e.g. Leontini, Thompson \& Hourigan 2006; Prasanth \& Mittal 2008).

In the absence of rotation $(\alpha=0)$, the cylinder response peaks near $U^{\star}=3$, with amplitudes close to $1.5 \times 10^{-3} \mathrm{D}$ and dominant frequencies close to twice the Strouhal frequency (dashed-dotted line in figure $5 b$ ). These vibrations correspond to the VIV previously observed at higher Reynolds numbers for non-rotating circular cylinders 
allowed to move in the in-line direction only (e.g. Okajima et al. 2002; Cagney \& Balabani 2013). The differences which can be noted in the shape and magnitude of the present response compared to these prior works are expected due to the difference in Reynolds number. The strong influence of $R e$ on in-line VIV amplitude was emphasized in a previous study concerning flexible cylinders (Bourguet, Karniadakis \& Triantafyllou 2011), and oscillation amplitudes comparable to those observed here were reported by Prasanth \& Mittal (2008) for a cylinder free to vibrate in both directions at similar $R e$.

A small peak of vibration amplitude persists close to $U^{\star}=3$ when the cylinder is subjected to a forced rotation at moderate values of $\alpha$. However, larger oscillations develop in a higher range of $U^{\star}$. The first vibration region extends beyond the above mentioned critical rotation rate of $\alpha \approx 1.8$, since the cylinder exhibits significant responses for $\alpha=2$. The bell-shaped trend of the response amplitudes occurring around $U^{\star}=6$ for $\alpha \leqslant 2$ and the fact that these vibrations appear close to the Strouhal frequency (dashed line in figure $5 b$ ) suggest that they are driven by a lock-in mechanism at the vortex shedding frequency, instead of twice the shedding frequency as in the non-rotating cylinder case. Such switch in the response dominant frequency was previously noted for non-axisymmetric cross-sections (Naudascher 1987). It relates to the alteration of the anti-symmetric nature of the wake and to the associated modification of the in-line forcing frequency. The link between the body response, the wake and the fluid forces is addressed in the next sections. Within this first vibration region, the oscillations of the rotating cylinder may reach $0.06 \mathrm{D}$ approximately around $U^{\star}=6$ for $\alpha=1.5$, which represents a considerable amplification compared to the non-rotating body case. In a narrow range around $U^{\star}=3$ and for low rotation rates $(\alpha \leqslant 1)$, two vibration components of comparable spectral amplitudes appear at a frequency close to the Strouhal frequency (fundamental component, $f_{x}$ ) and at twice this frequency (second harmonic component, $2 f_{x}$ ). In this zone, the second harmonic component may dominate the response. Except in this limited zone of the parameter space, the cylinder oscillation remains close to sinusoidal. It is dominated by its fundamental frequency, with very small higher harmonic contributions. No structural oscillation occurs for $\alpha=2.5$ and $\alpha=2.625$.

Much larger oscillation amplitudes are encountered in the second vibration region located in the higher range of $\alpha$. For $\alpha=2.7$, the response is characterized by a bell shape centred about $U^{\star}=17$. For $\alpha \geqslant 2.75$, the cylinder starts vibrating around $U^{\star}=8$ and the oscillation amplitude does not return to zero as $U^{\star}$ increases. In spite of some undulations in its evolution, the amplitude globally increases with $U^{\star}$ and it reaches more than 2.5 body diameters for $\left(\alpha, U^{\star}\right)=(3.5,23)$. For a given reduced velocity, the amplitude tends to increase with $\alpha$ in this second vibration region. It can be noted that the bell shape of the response for $\alpha=2.7$ is continuously prolongated for $\alpha=2.75$ by a well-defined bump in the response curve around $U^{\star}=17$. It illustrates the onset of the second oscillatory regime, at the lower edge of this vibration region. In the second vibration region, the oscillation is still dominated by its fundamental frequency component (and thus close to sinusoidal) but the fundamental frequency is always much smaller than the Strouhal frequency (dashed line in figure $6 b$ ) and lower than the oscillator natural frequency $\left(f^{\star}<1\right)$, in contrast with the response frequencies identified in the first vibration region (figure $5 b$ ). The modulations of the dominant vibration frequency are connected to the variability of the effective added mass, as discussed in $\S 5$. The responses occurring for $\alpha \geqslant 2.75$ resemble the galloping oscillations reported for non-axisymmetric bodies (e.g. Bearman et al. 1987; Nemes et al. 2012). Such responses were previously observed for a rotating circular cylinder 
free to oscillate in both the in-line and cross-flow directions (Stansby \& Rainey 2001; Yogeswaran \& Mittal 2011) but not when the body was restrained to a single degreeof-freedom in the cross-flow direction (Bourguet \& Lo Jacono 2014). Due to their evolution as functions of $U^{\star}$, the responses encountered in the second vibration region are referred to as galloping-like oscillations in the following. It should however be mentioned that these vibrations cannot be predicted through quasi-steady analysis, as shown in appendix A.

The oscillatory responses obtained through two- and three-dimensional simulations are identical at $\alpha=2.75$ and minor differences can be noted at higher rotation rates (circled blue symbols in figure 6). The vibration amplitudes obtained through three-dimensional simulation are slightly smaller. However, the impact of the flow three-dimensionality on the response remains limited and, as also observed for the time-averaged displacement, the trends identified on the basis of the two-dimensional results are essentially confirmed by the three-dimensional ones.

The above analysis reveals the existence of two distinct vibration regions in the $\left(\alpha, U^{\star}\right)$ parameter space. In the first vibration region, up to a rotation rate close to 2 , the cylinder exhibits low-amplitude vibrations with characteristics typical of VIV regime (trend as a function of $U^{\star}$, frequency), and whose largest magnitudes appear around $U^{\star}=6$. In the second vibration region, beyond $\alpha=2.7$ and $U^{\star}=8$ approximately, galloping-like responses of large amplitudes develop. No vibration is observed between these two regions. The present results also indicate that the flow three-dimensional transition occurs within the second vibration region. These aspects are further studied in the next section which clarifies the properties of the flow downstream of the freely vibrating body.

\section{Flow physics}

The occurrence of structural oscillations that resemble both VIV and galloping responses, within two distinct regions of the parameter space under study, raises the question of the synchronization between the oscillation of the rotating cylinder and the flow unsteadiness in its wake. This point is addressed as a first step in §4.1. The independent effects of rotation and oscillation on the vortex shedding pattern downstream of a circular cylinder have been well documented in the literature (e.g. Williamson \& Roshko 1988; Badr et al. 1990; Brika \& Laneville 1993; Govardhan \& Williamson 2000; Stojković et al. 2002; Mittal \& Kumar 2003; Carberry, Sheridan \& Rockwell 2005). In a recent work (Bourguet \& Lo Jacono 2014), novel flow patterns were observed in the wake of a rotating cylinder freely vibrating in the cross-flow direction, such as, for instance, an asymmetric pattern composed of a triplet of vortices and a single vortex shed per oscillation cycle ( $\mathrm{T}+\mathrm{S}$ pattern). The flow structures developing in the present configuration, within each response region, remain to be studied. They are examined as a second step in $\S 4.2$, with special attention paid to the appearance of new patterns. Finally, the three-dimensional transition identified in the range of high rotation rates in the previous section is investigated in $\S 4.3$.

\subsection{Wake-body synchronization}

The frequency content of the flow is quantified via the PSD of the time series of the cross-flow component of the flow velocity $(v)$ at a point located at $(x / D, y / D)=$ $(10,0)$, i.e. $10 D$ downstream of the cylinder position in quiescent fluid. The time series of $v$ is periodic in all studied cases. The PSD of $v$ is plotted as a function of 
the reduced velocity, for each rotation rate, in figure 7. The steady flow cases where no vibration is observed $(\alpha=2.5$ and $\alpha=2.625)$ are not considered here. At each reduced velocity, the PSD is normalized by the magnitude of the largest peak. As reported in $\S 3.2$, the cylinder vibration is generally close to sinusoidal and dominated by its fundamental frequency $\left(f=f_{x}\right)$, except for $\alpha=0.5$ around $U^{\star}=3$, where the second harmonic component dominates $\left(f=2 f_{x}\right)$. In figure 7 , the dominant frequency of the cylinder vibration is denoted by a red triangle and the fundamental vibration frequency, when it differs from the dominant frequency, is indicated by a blue cross $\left(U^{\star}=2.75\right.$ and $U^{\star}=3$ for $\alpha=0.5$, figure $7 b$ ). The dominant vibration frequency is simply referred to as the vibration frequency or response frequency in the following.

Within the first vibration region $(\alpha \leqslant 2)$, the PSD in figure $7(a-e)$ show that for each rotation rate and reduced velocity, the vibration frequency coincides with the fundamental frequency of the flow velocity or twice this frequency. The bell-shaped structural responses occurring in this region (figure 5) thus develop under a condition of wake-body synchronization or lock-in, which is the central mechanism driving non-rotating cylinder VIV. This confirms the similarity between the responses encountered in this area of the parameter space and VIV. It can be noted that the very low-amplitude oscillations appearing away from the interval of $U^{\star}$ associated with the peak amplitudes of response are also synchronized with the wake, at a frequency close to the Strouhal frequency (or twice this frequency), as previously observed for non-rotating cylinders (e.g. Prasanth \& Mittal 2008). The alteration of the wake anti-symmetry induced by the rotation is accompanied, within the first vibration region, by a switch of the vibration frequency, generally equal to the fundamental wake frequency for $\alpha>0$ versus twice this frequency for $\alpha=0$. Dominant vibration components at twice the fundamental wake frequency may still appear for $\alpha=0.5$, under vortex shedding patterns that remain close to, even though not exactly, anti-symmetric. The wake patterns are analysed in more details in the next subsection.

A striking phenomenon is that the condition of wake-body synchronization persists in the second vibration region $(\alpha \geqslant 2.7$, figure $7 f-j)$, where the rotating cylinder exhibits galloping-like responses (figure 6). Prior works have shown that galloping oscillations do not generally involve such synchronization mechanism (e.g. Parkinson \& Smith 1964; Païdoussis et al. 2010). Synchronized states leading to steps in the evolution of the response amplitude as a function of the reduced velocity were previously reported over short intervals of $U^{\star}$ (Zhao et al. 2014a); they explain the 'kinks' identified in the galloping response curve by Bearman et al. (1987). These observations however differ from the present results where the vibration frequency is found to be equal to the fundamental wake frequency for all $U^{\star}$ in this range of $\alpha$. It is recalled that the term galloping-like is used in this paper in reference to the trend of the response as a function of the reduced velocity (i.e. amplitude increasing unboundedly with $U^{\star}$ ). The large-amplitude oscillations occurring in the second vibration region are not driven by a quasi-steady mechanism (appendix A) and appear to be systematically associated with wake-body synchronization.

The successive emergence of large higher harmonic contributions in the flow velocity spectrum, as $U^{\star}$ increases within the second vibration region, suggests that an increasing number of vortices are shed per oscillation cycle. This point is examined in the following. The three-dimensional simulation results corroborate the above mentioned findings. 


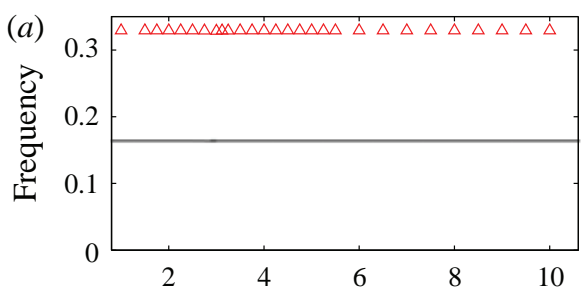

(b)
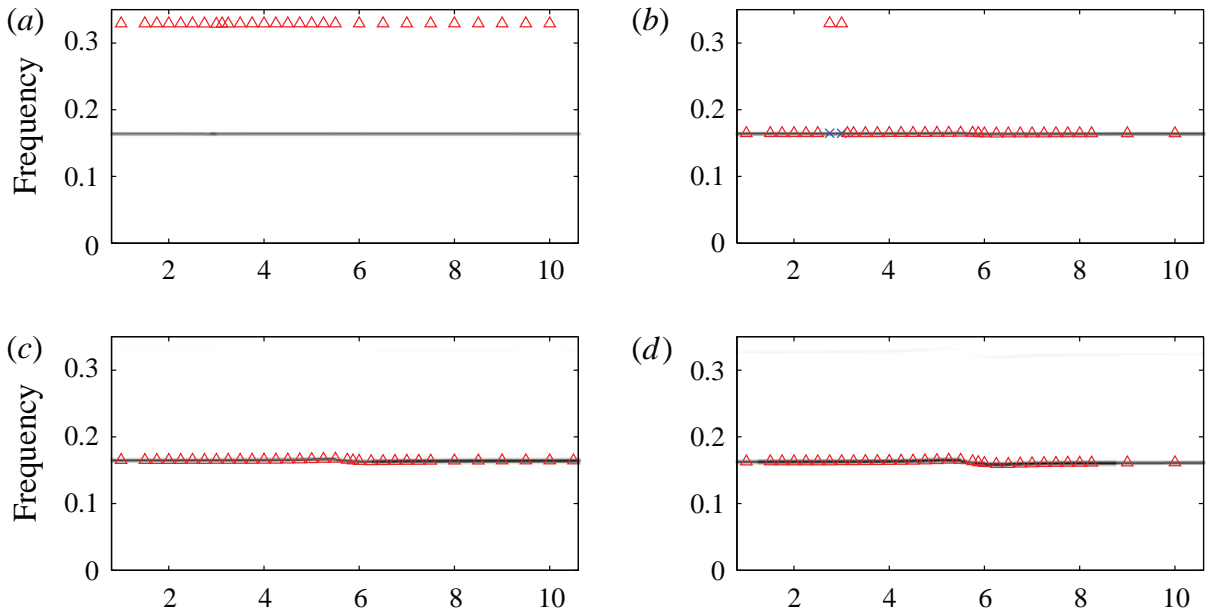

$(d)$
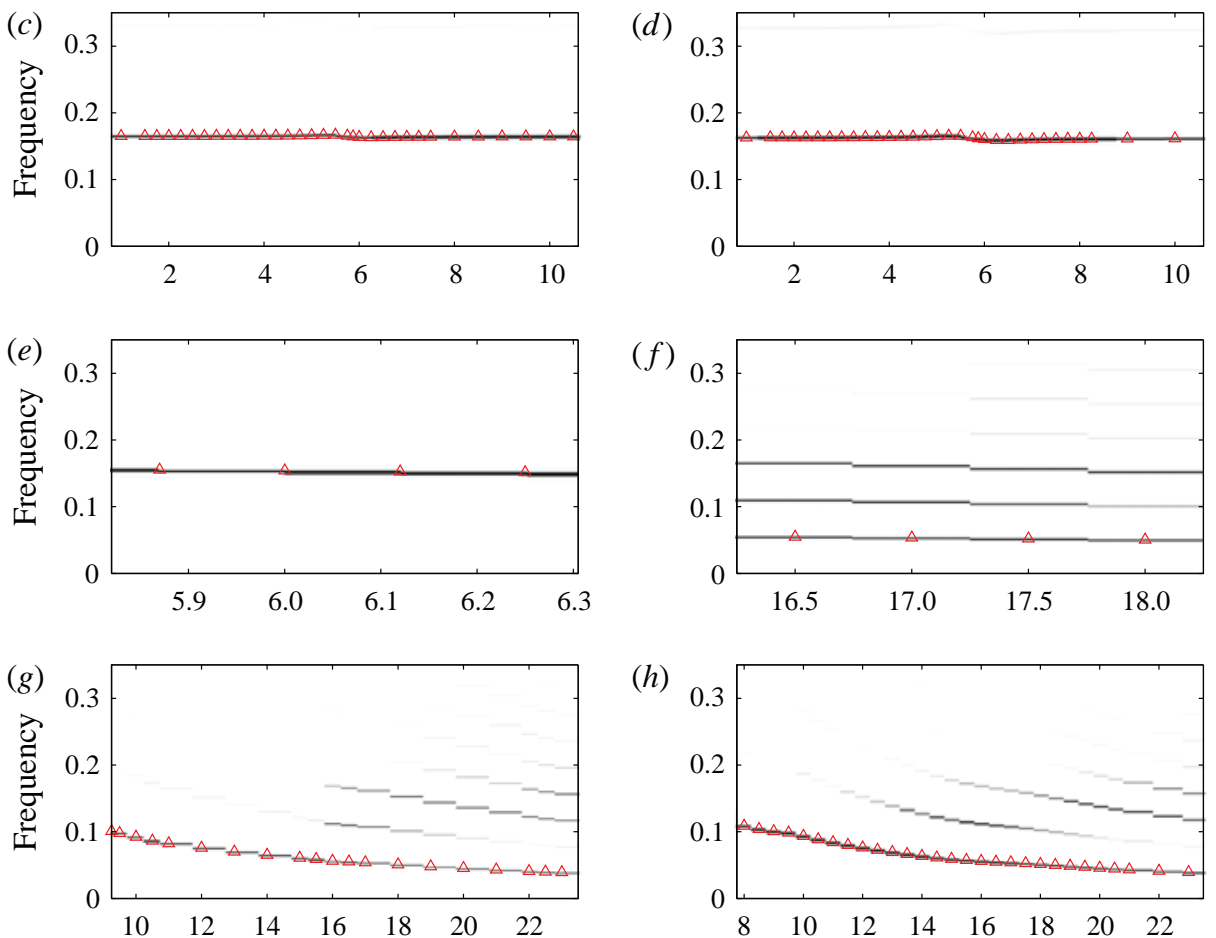

(h)
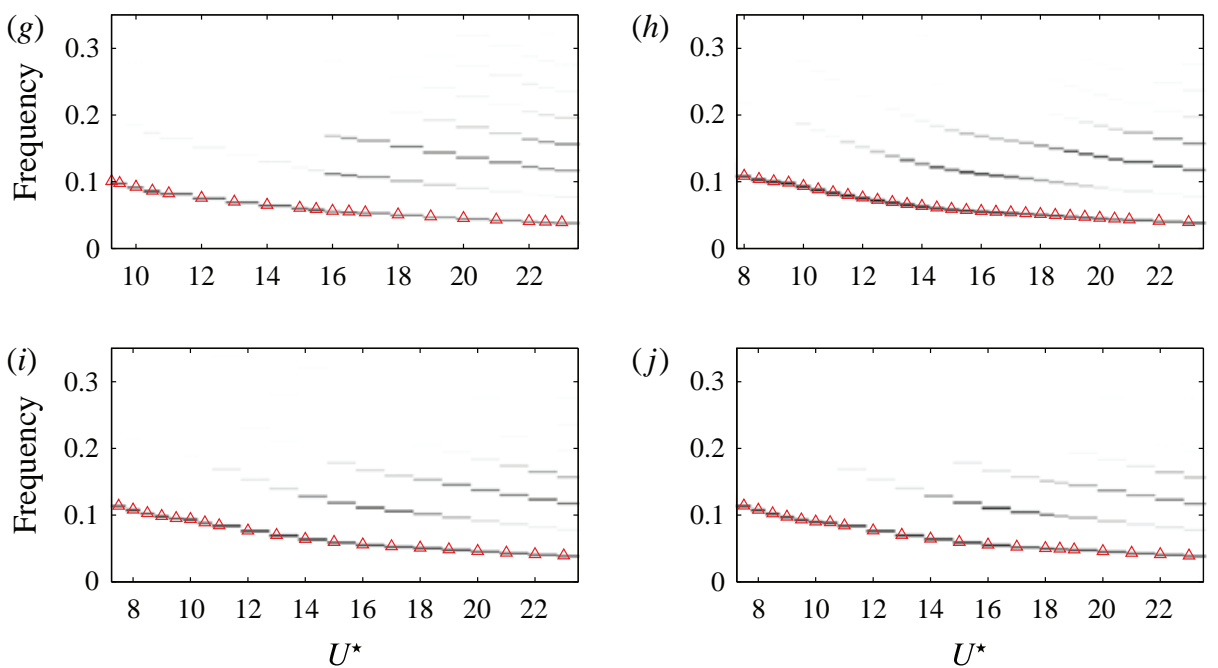

FIgURE 7. (Colour online) PSD of the cross-flow component of the flow velocity at $(x / D, y / D)=(10,0)$ as a function of the reduced velocity for $(a) \alpha=0,(b) \alpha=0.5,(c)$ $\alpha=1,(d) \alpha=1.5,(e) \alpha=2,(f) \alpha=2.7,(g) \alpha=2.75,(h) \alpha=3,(i) \alpha=3.25,(j) \alpha=3.5$. For each reduced velocity, the PSD is normalized by the magnitude of the largest peak. The colour levels range from 0 (white) to 1 (black). The dominant vibration frequency of the cylinder $(f)$ is indicated by a red triangle and the fundamental vibration frequency $\left(f_{x}\right)$, when it differs from the dominant frequency, is denoted by a blue cross. 
(a)

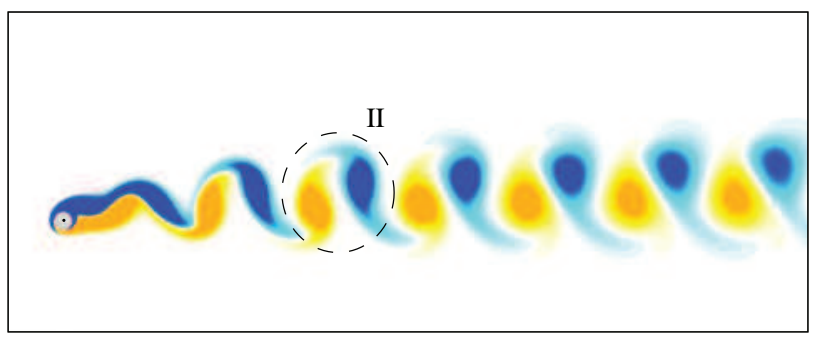

(b)

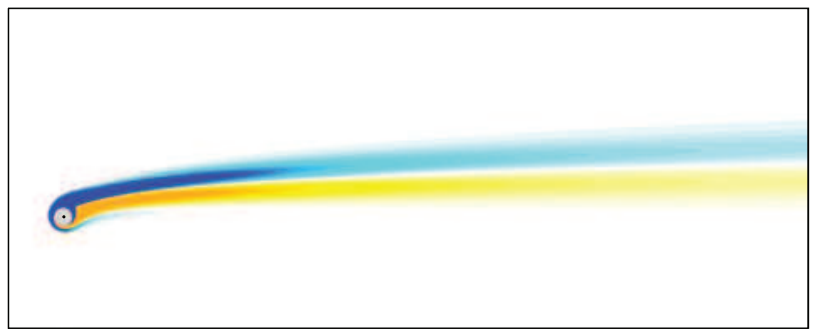

FIGURE 8. (Colour online) Instantaneous iso-contours of spanwise vorticity: (a) II pattern, $\left(\alpha, U^{\star}\right)=(1.5,1), \omega_{z} \in[-0.3,0.3] ;(b) \mathrm{D}^{+}$pattern, $\left(\alpha, U^{\star}\right)=(2.5,1), \omega_{z} \in[-0.2,0.2]$. Positive/negative vorticity values are plotted in yellow/blue. A dashed line indicates the vortices formed over one shedding cycle. Part of the computational domain is shown.

\subsection{Wake patterns}

The boundaries of the steady and unsteady flow areas of the $\left(\alpha, U^{\star}\right)$ domain coincide with the limits of the two vibration regions identified in figure $4(b)$. A variety of unsteady flow patterns are encountered in the parameter space under study. A nomenclature based on the number of vortices shed per fundamental period of the flow unsteadiness (based on the fundamental frequency of $v$ at $(x / D, y / D)=(10,0))$ is adopted to designate the different wake patterns. For instance, the II pattern is associated with the formation of two vortices per cycle while the $\mathrm{V}$ pattern corresponds to the formation of five vortices. Due to the condition of wake-body synchronization identified in $\S 4.1$, the present nomenclature indicates the number of vortices shed per oscillation period, once the body vibrates, except for the low rotation rates (e.g. $\alpha=0$ and some values of $U^{\star}$ for $\alpha=0.5$ ) where the cylinder may oscillate at twice the fundamental frequency of the wake. In the present configuration where body rotation results in asymmetric patterns and substantial deviations compared to the patterns previously reported in the absence of rotation (Williamson \& Roshko 1988), this nomenclature is preferred to the more usual approach based on the identification of single vortices $(\mathrm{S})$ and pairs $(\mathrm{P})$ or triplets $(\mathrm{T})$ of vortices. It may appear as a simpler way to describe the vortical structures in the context of this study, as the distinction between the $2 \mathrm{~S}$ and $\mathrm{P}$ topologies, for example, may be subtle.

The different wake patterns are illustrated in figures 8-10 by instantaneous iso-contours of spanwise vorticity, for selected values of the couple $\left(\alpha, U^{\star}\right)$ which are designated by black dots in figure 4(b). In figures 8-10, a dashed line regroups the vortices formed over one shedding cycle, the corresponding pattern is mentioned and the in-line trajectory of the body centre is indicated by a segment.

When the cylinder does not vibrate (or exhibits vibrations of negligible amplitudes, e.g. for $U^{\star}<2$ ), two wake patterns develop in the range of $\alpha$ investigated. For $\alpha<1.8$, 
(a)

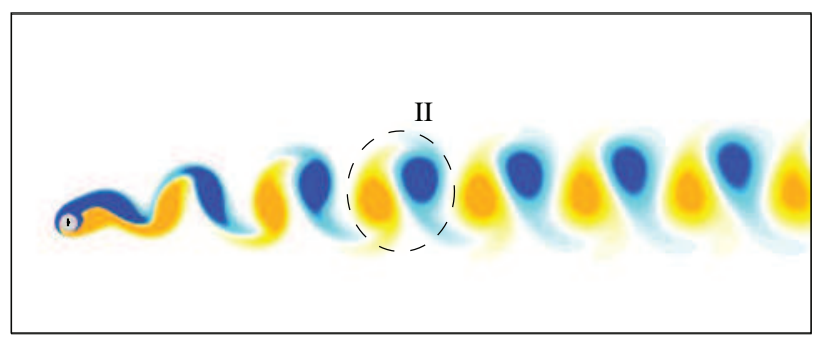

(b)

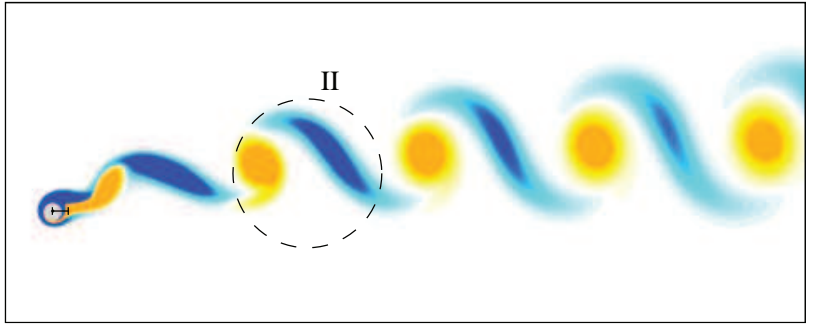

(c)

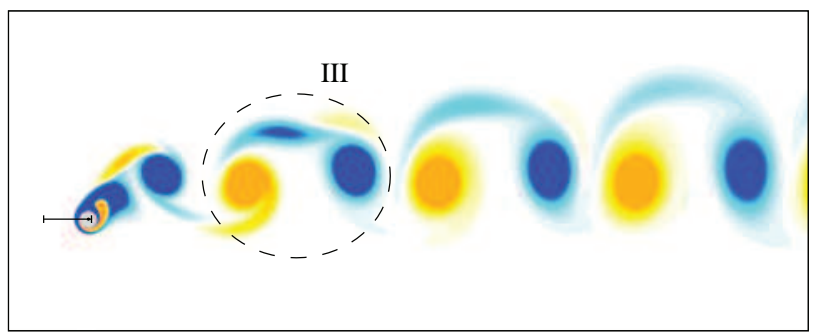

FIgURE 9. (Colour online) Instantaneous iso-contours of spanwise vorticity: (a) II pattern, $\left(\alpha, U^{\star}\right)=(1.5,5.875), \omega_{z} \in[-0.3,0.3] ;(b)$ II pattern, $\left(\alpha, U^{\star}\right)=(3.25,8), \omega_{z} \in$ $[-0.25,0.25]$; $(c)$ III pattern, $\left(\alpha, U^{\star}\right)=(3.25,10.5), \omega_{z} \in[-0.3,0.3]$. Positive/negative vorticity values are plotted in yellow/blue. A dashed line indicates the vortices formed over one shedding cycle. The trajectory of the cylinder centre is indicated by a segment. Part of the computational domain is shown.

the wake is characterized by the formation of two counter-rotating vortices per cycle (II or $2 \mathrm{~S}$ pattern), as in the stationary cylinder case (figure $8 a$ ). As also noted in previous studies (e.g. Mittal \& Kumar 2003), the rotation induces an asymmetry in the strength of the positive and negative vortices, and an upward deviation of the wake. Beyond $\alpha=1.8$, the wake of the non-vibrating cylinder is composed of two elongated layers of vorticity of opposite signs and deflected upwards (figure $8 b$ ); the flow is steady. In a prior work (Bourguet \& Lo Jacono 2014) this pattern was named $\mathrm{D}^{+}$, in reference to the positive value of the associated drag force, which becomes negative at higher rotation rates.

The occurrence of free structural oscillations is accompanied by a local extension of the unsteady flow region located in the lower range of $\alpha$, up to $\alpha=2$ approximately around $U^{\star}=6$. It is also associated with the appearance of a second unsteady flow area, which corresponds to the second vibration zone in the higher range of $\alpha$.

Within the first unsteady flow region $(\alpha \leqslant 2)$, the wake is still characterized by the II pattern once the body exhibits significant vibrations (figure $9 a$ ). 
(a)

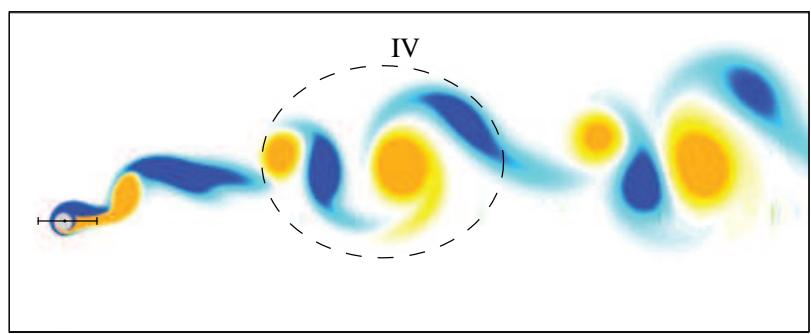

(b)

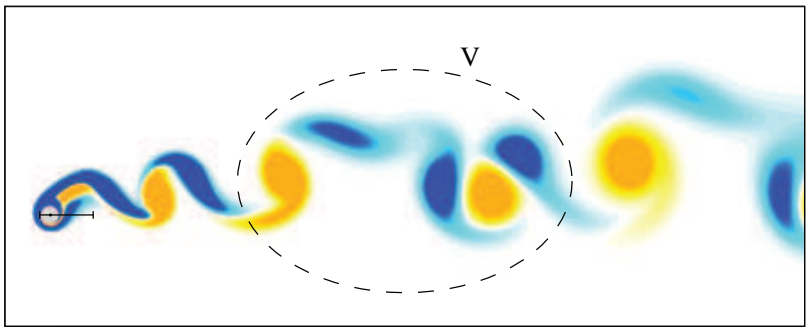

(c)

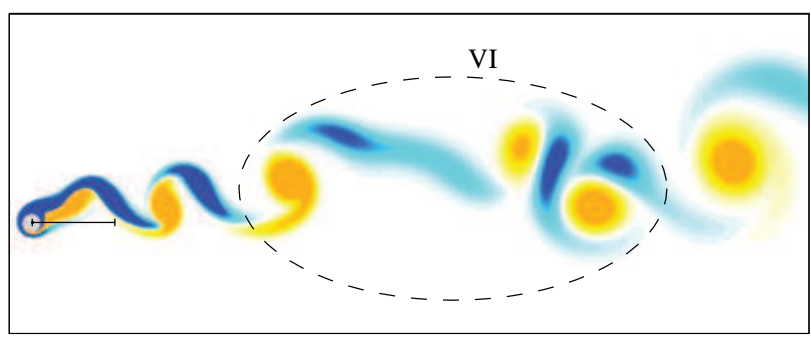

FIGURE 10. (Colour online) Instantaneous iso-contours of spanwise vorticity: (a) IV pattern, $\left(\alpha, U^{\star}\right)=(3.5,15), \omega_{z} \in[-0.2,0.2] ;(b) \mathrm{V}$ pattern, $\left(\alpha, U^{\star}\right)=(2.75,18), \omega_{z} \in$ $[-0.2,0.2] ;(c)$ VI pattern, $\left(\alpha, U^{\star}\right)=(3,23), \omega_{z} \in[-0.3,0.3]$. Positive/negative vorticity values are plotted in yellow/blue. A dashed line indicates the vortices formed over one shedding cycle. The trajectory of the cylinder centre is indicated by a segment. Part of the computational domain is shown.

A detailed map of the wake patterns occurring in the second vibration region is presented in figure 11. The cases visualized in figures 9 and 10 are indicated by black dots. It is recalled that the limits of the different zones remain approximate. The formation of two vortices per cycle is also observed in the second unsteady flow region, as illustrated in figure $9(b)$. Within this second region, for $\alpha>2.85$ approximately, the number of vortices shed per oscillation period tends to increase with $U^{\star}$ and up to six vortices may form beyond $U^{\star}=20$ (VI pattern, figure $10 \mathrm{c}$ ). The existence of these wake patterns and their location in the parameter space are confirmed by the three-dimensional simulation results, especially beyond the critical rotation rate associated with the flow three-dimensional transition, as shown in §4.3. By adopting the more usual nomenclature, the $\mathrm{V}$ and VI patterns may, for instance, be referred to as $\mathrm{T}+\mathrm{P}$ and $\mathrm{Q}+\mathrm{P}$, respectively ( $\mathrm{Q}$ would designate a quartet of vortices). These flow topologies did not appear in the wake of a rotating cylinder freely oscillating in the cross-flow direction within a comparable parameter space (Bourguet \& Lo Jacono 2014). To the authors' knowledge, such asymmetric multi-vortex patterns were not previously reported. 


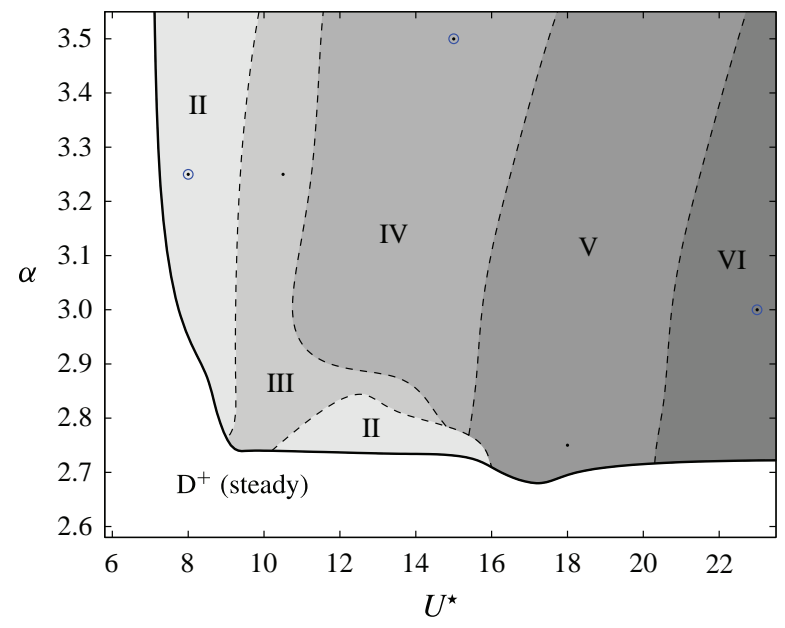

FIGURE 11. (Colour online) Wake pattern as a function of the rotation rate and reduced velocity in the second vibration region. The limit of the unsteady flow region, which coincides with the vibration region, is indicated by a plain line. Black dots denote the cases considered in figures 9 and 10 (two-dimensional results) while blue circles designate the cases presented in figures 13 and 14 (three-dimensional results).

Prior works concerning oscillating non-rotating cylinders have emphasized the close connection between wake pattern selection and oscillation amplitude and frequency (e.g. Williamson \& Roshko 1988). The pattern selected in the present configuration for each couple $\left(\alpha, U^{\star}\right)$ is indicated in the rotation rate-response amplitude-frequency domain in figure $12(a)$ and the results obtained for all the rotation rates are gathered in the response amplitude-frequency domain in figure $12(b)$. The first vibration region, where the oscillation amplitude remains lower than $0.1 D$ and which is characterized by a single flow topology (II pattern) is not considered here. The analysis concerns the second vibration region where five distinct patterns are encountered. Results obtained from the additional two-dimensional simulations performed to determine the maps in figures $4(b)$ and 11 , as well as three-dimensional simulation results (denoted by circled blue symbols) are included in figure 12(b). As a global trend, also noted for a rotating cylinder oscillating in the cross-flow direction (Bourguet \& Lo Jacono 2014), the number of vortices shed per cycle tends to increase with the vibration amplitude and as the frequency decreases. In some cases, a sudden change in the response amplitude can be directly associated with an alteration of the wake pattern, as, for instance, between $\left(\alpha, U^{\star}\right)=(2.75,15.5)$ and $\left(\alpha, U^{\star}\right)=(2.75,16)$ where the amplitude increases from $0.4 D$ to $1.3 D$, around $f=0.06$, while the flow topology switches between patterns II and V. However, it should be mentioned that the transitions between successive patterns cannot be systematically related to the undulations noted in the evolution of the vibration amplitude.

The next subsection focuses on the occurrence of the three-dimensional transition in the flow past the freely oscillating rotating cylinder.

\subsection{Three-dimensional transition}

The impact of a forced rotation on the flow three-dimensional transition was highlighted in previous studies concerning rigidly mounted cylinders (e.g. Mittal 2004; 


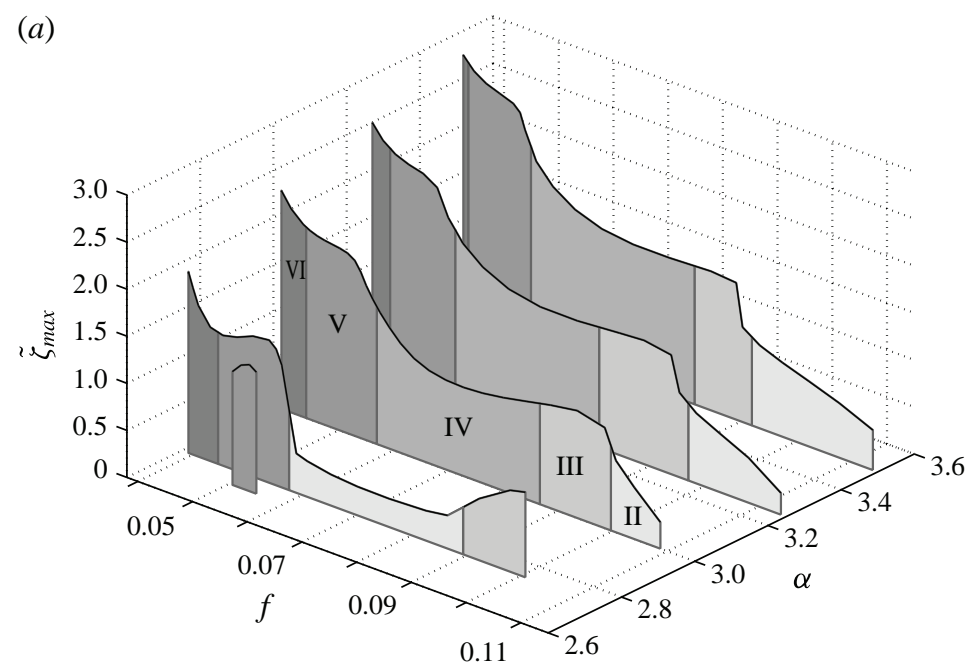

(b)

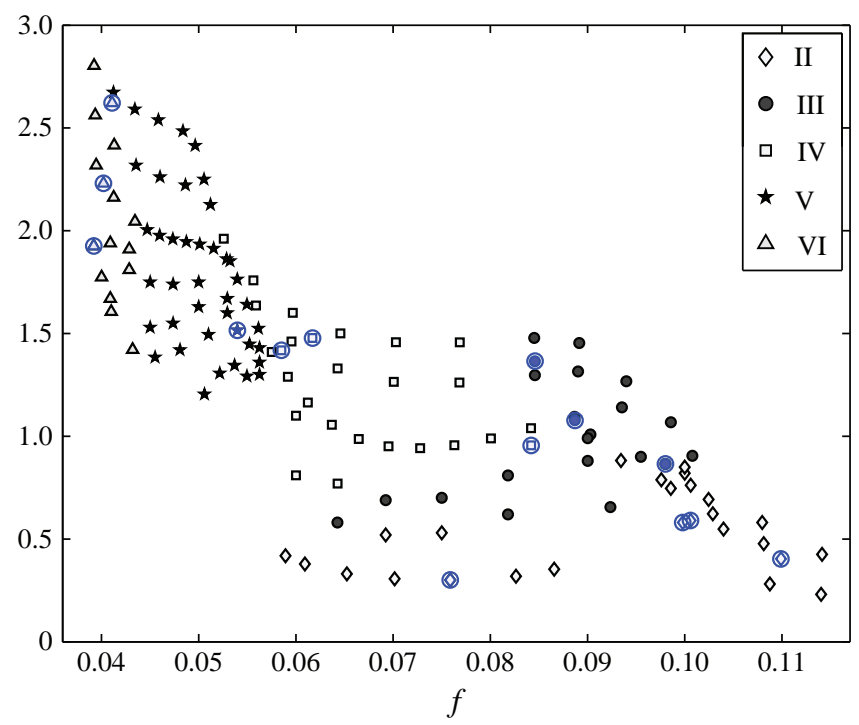

FIGURE 12. (Colour online) Wake pattern as a function of (a) the rotation rate, maximum amplitude of vibration and vibration frequency and $(b)$ the maximum amplitude of vibration and vibration frequency for all the rotation rates $\alpha \geqslant 2.7$. In $(a)$, the colour code used to indicate the type of wake pattern is the same as in figure 11. In (b), the three-dimensional simulation results are denoted by circled blue symbols.

Radi et al. 2013). At $R e=100$, the three-dimensional transition was found to appear for $\alpha \approx 3.7$ in this case (Pralits et al. 2013; Rao et al. 2013a). It was also shown that the flow may remain two-dimensional beyond this critical rotation rate when the cylinder is subjected to transverse oscillations (Bourguet \& Lo Jacono 2014). The above mentioned critical value is larger than the maximum rotation rate considered in the present work $(\alpha=3.5)$. However, as reported in $\S 3$ (e.g. figure 6), the structural responses predicted by two- and three-dimensional simulations are identical for $\alpha=2.75$ over a range of $U^{\star}$ values that encompasses the entire vibration window 
(a)

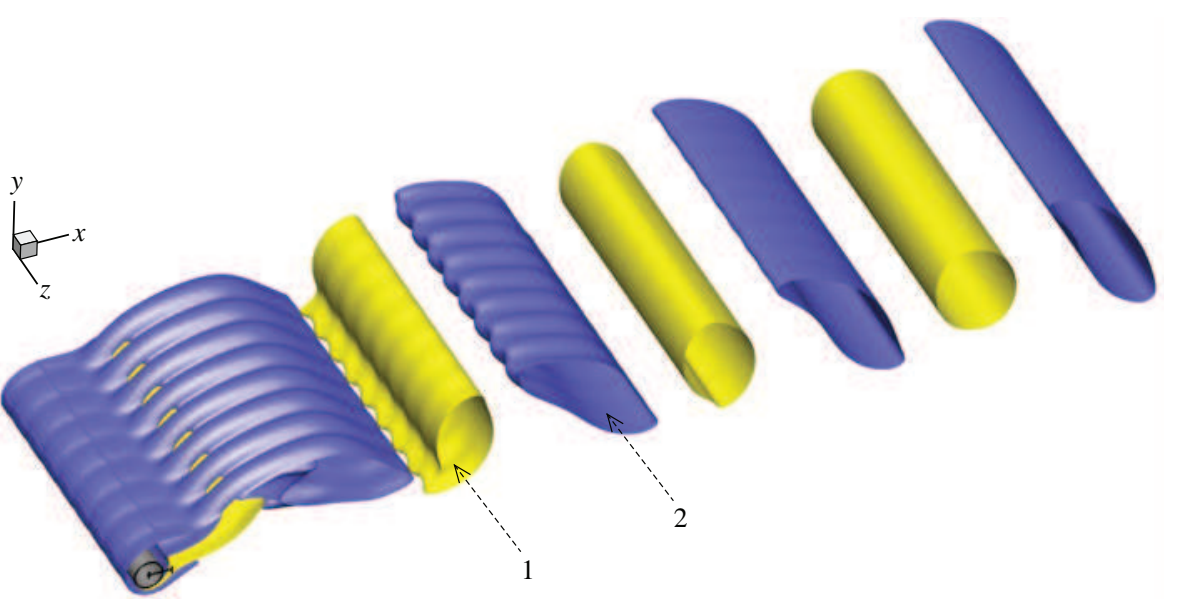

(b)

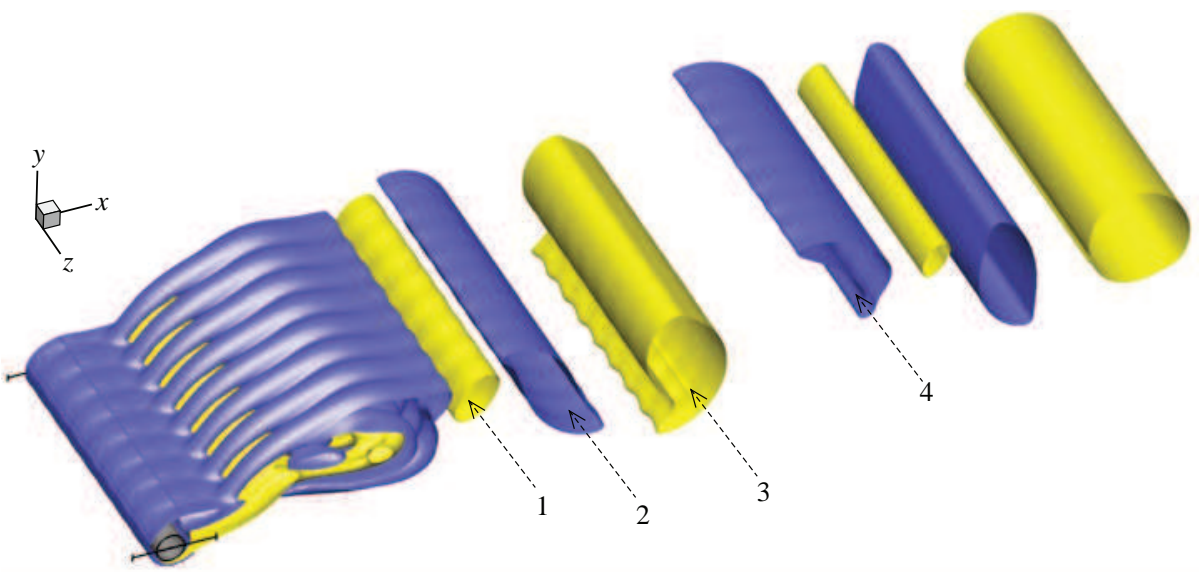

(c)

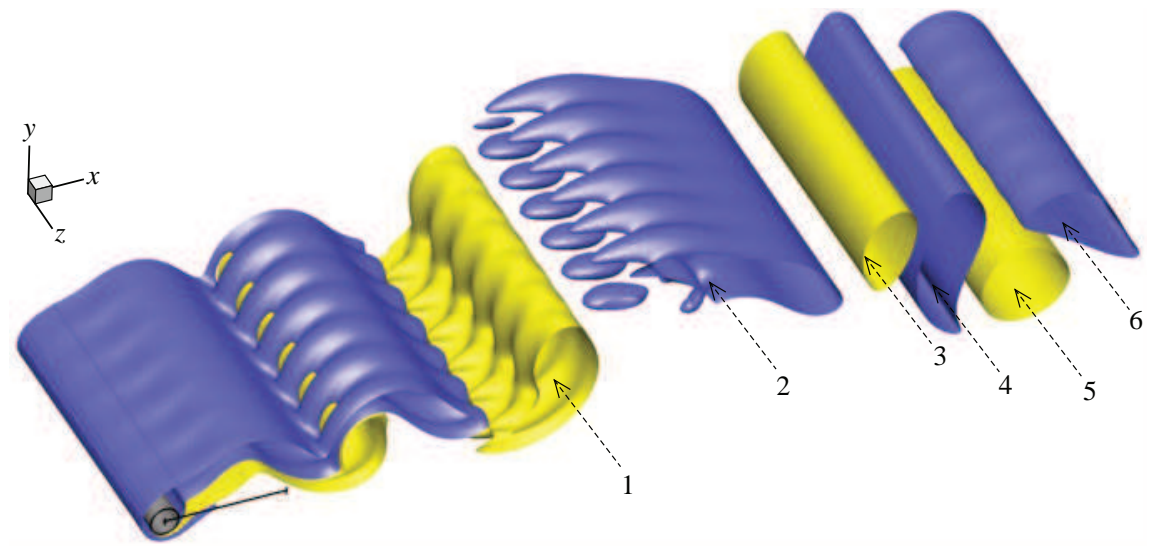

FIGURE 13. (Colour online) Instantaneous iso-surfaces of spanwise vorticity (threedimensional simulations, $\left.\omega_{z}= \pm 0.07\right)$ : (a) II pattern, $\left(\alpha, U^{\star}\right)=(3.25,8)$; (b) IV pattern, $\left(\alpha, U^{\star}\right)=(3.5,15)$; (c) VI pattern, $\left(\alpha, U^{\star}\right)=(3,23)$. Positive/negative vorticity values are plotted in yellow/blue. Dashed arrows indicate the vortices shed over one cycle. The trajectory of the cylinder centre is indicated by a segment. Part of the computational domain is shown. 
(a)

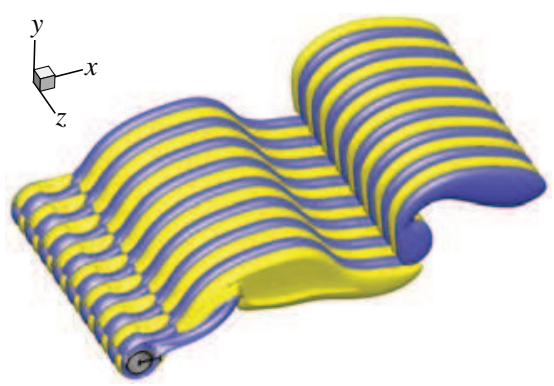

(b)

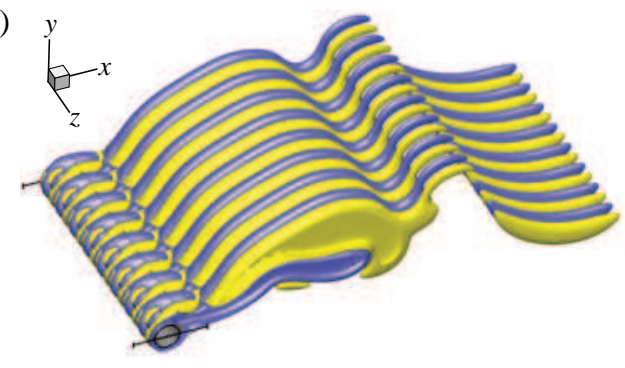

(c)

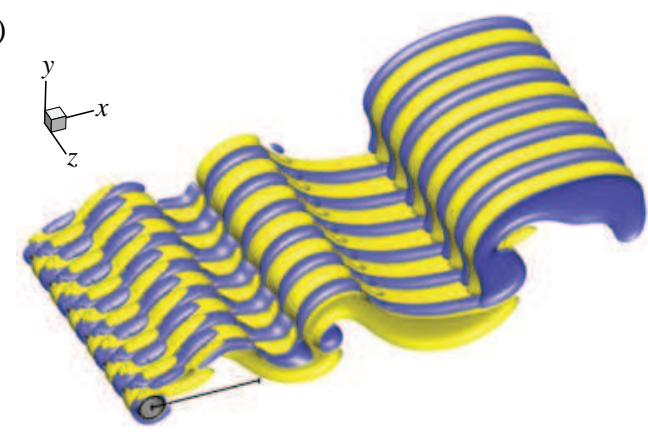

FIgURE 14. (Colour online) Same as figure 13 for the streamwise vorticity $\left(\omega_{x}= \pm 0.01\right)$.

up to $U^{\star}=23$, but they systematically differ for $\alpha=3$. Therefore, the flow past the cylinder oscillating in the in-line direction undergoes three-dimensional transition in the second vibration region for a critical rotation rate located between 2.75 and 3 , i.e. substantially lower than in the absence of vibration. As a consequence, while the onset of flow three-dimensionality is delayed under cross-flow oscillation, it tends to occur earlier under in-line oscillation. The in-line motion causes large variations of the instantaneous rotation rate and Reynolds number based on the instantaneous flow velocity in the cylinder frame. During an oscillation cycle, the instantaneous rotation rate and Reynolds number may reach the critical values associated with the three-dimensional transition in the rigidly mounted cylinder configuration. This aspect is briefly addressed in appendix B but it appears that the occurrence of the three-dimensional transition in the vibrating cylinder case cannot be directly connected to the flow behaviour in the rigidly mounted body configuration. The results reported in the following do not aim at providing an extensive analysis of the transition mechanisms. Instead, the objective is to present some of the principal features of the three-dimensional flow within the parameter space under study and to clarify the potential alteration of the wake patterns identified in $\$ 4.2$.

Visualizations of the three-dimensional flow in selected points of the second vibration region (indicated by blue circles in figures $4 b$ and 11) are presented in figures 13 and 14, by means of instantaneous iso-surfaces of the spanwise and streamwise vorticity components, respectively. In these plots, a segment indicates the trajectory of the cylinder centre. As illustrated in these figures, the flow three-dimensional transition is characterized by a regular spanwise undulation particularly pronounced around the cylinder and in the near wake, and which tends to vanish further downstream, where the spanwise vortex rows appear essentially two-dimensional. Among all simulated cases, the spanwise wavelength of the 
three-dimensional pattern is found to range between $1 D$ and $1.7 D$, approximately. A typical wavelength close to $1.7 D$ was previously identified by means of direct numerical simulation at the onset of the three-dimensional transition, for a rigidly mounted rotating cylinder at $R e=100$ (Bourguet \& Lo Jacono 2014). Comparable wavelengths were reported in this case on the basis of linear stability analyses (Pralits et al. 2013; Rao et al. 2013a). In the present configuration, the spanwise wavelength may exhibit significant variations for a given rotation rate, depending on the reduced velocity. For instance, for $\alpha=3$, wavelengths approximately equal to $1.25 D, 1.1 D, 1 D$ and $1.7 D$ are identified for $U^{\star} \in\{9,11,15.5,23\}$. It can be noted that the evolution of the wavelength is not monotonic as a function of $U^{\star}$ and no evident connection appears with the vibration amplitude and frequency. A comparable variability of the spanwise wavelength is observed as a function of $\alpha$, for a fixed value of $U^{\star}$.

Comparisons of the flow structures obtained through two- and three-dimensional simulations for the same couples $\left(\alpha, U^{\star}\right)$ indicate that, across the entire parameter space investigated, the slight spanwise modulations associated with the threedimensional transition do not significantly alter the shape of the wake patterns reported in $\S 4.2$, under two-dimensional flow assumption. The absence of substantial impact of the three-dimensional transition on the wake patterns is illustrated in figure 13 where the II, IV and VI patterns, previously depicted on the basis of two-dimensional simulation results in figures $9(b), 10(a)$ and $(c)$, respectively, can be clearly identified. In this figure the spanwise vortices shed over one oscillation cycle are indicated by dashed arrows. The three-dimensional nature of the flow has only a limited influence on the structural responses: as shown in $\S 3$, the vibration amplitudes and frequencies obtained from the three-dimensional simulations performed beyond the critical rotation rate remain close to those predicted under two-dimensional flow assumption.

In order to complement the investigation of the flow-structure system behaviour in each vibration region, an analysis of the forces exerted by the fluid on the flexibly mounted rotating cylinder, including a quantification of the effect of the three-dimensional transition, is presented in the next section.

\section{Fluid forces}

Statistical and spectral analyses of the fluid forces are reported in $\S 5.1$. The phasing mechanisms between force and displacement, and the effective added mass induced by the fluid are examined in $\S 5.2$, with an emphasis on the differences occurring between the two vibration regions.

\subsection{Statistics and spectral analysis}

The time-averaged in-line and cross-flow force coefficients are plotted as functions of the reduced velocity in figures 15 and 16. For each rotation rate, the values of $\bar{C}_{x}$ and $\bar{C}_{y}$ in the rigidly mounted cylinder case are indicated by dashed lines. In the absence of vibration, $\bar{C}_{x}$ decreases as $\alpha$ increases within the range investigated, as also reported in previous works (Stojković et al. 2003; Bourguet \& Lo Jacono 2014). The global decrease of $\bar{C}_{y}$ as a function of $\alpha$ in the non-vibrating body case corresponds to the Magnus effect (Prandtl 1925). The negative sign is due to the counter-clockwise rotation. In agreement with prior studies concerning rigidly mounted cylinders (e.g. Mittal \& Kumar 2003), $\bar{C}_{y}$ differs from the prediction through potential flow theory $\left(C_{y}=-2 \pi \alpha\right)$ and exceeds the limit of $4 \pi$ proposed by Prandtl (1925). Substantial 


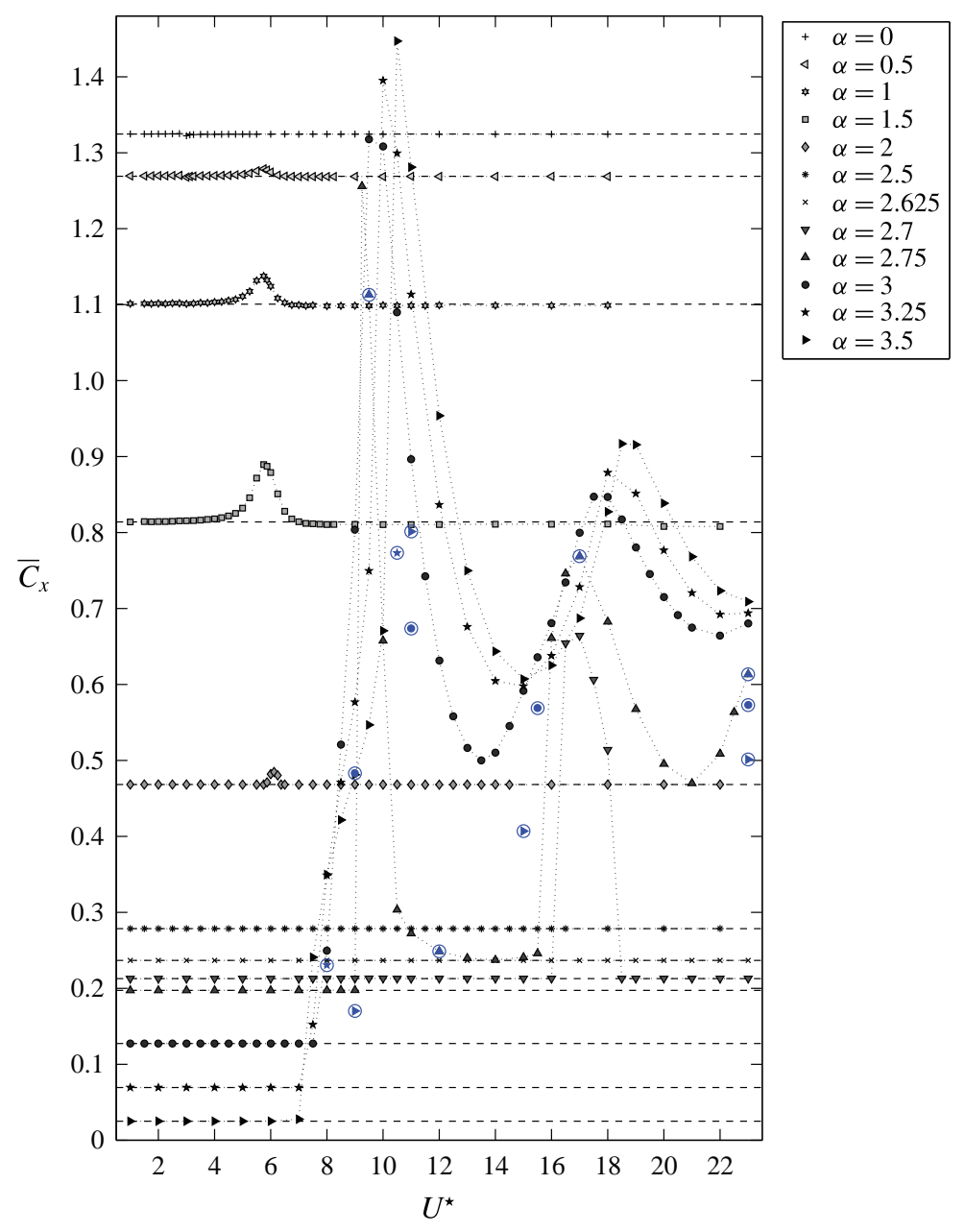

FIgURE 15. (Colour online) Time-averaged in-line force coefficient as a function of the reduced velocity. A dashed line indicates, for each rotation rate, the time-averaged force coefficient in the rigidly mounted cylinder case. The three-dimensional simulation results are denoted by circled blue symbols.

alterations of $\bar{C}_{x}$ and $\bar{C}_{y}$ can be observed when the cylinder oscillates compared to the rigidly mounted body case (dashed lines in figures 15 and 16).

The deviation of $\bar{C}_{x}$ from its value in the rigidly mounted body case is plotted as a function of the oscillation maximum amplitude in figure 17. For clarity, due to the difference in the typical oscillation amplitudes, the results corresponding to each vibration region are presented separately. A small reduction of $\bar{C}_{x}$ compared to the stationary body value may occur in the range of low oscillation amplitudes (e.g. for $\alpha=1.5$ ). However, except in this range and regardless of the type of wake pattern, the structural oscillations are generally associated with an amplification of $\bar{C}_{x}$. As also shown in previous studies concerning non-rotating cylinders oscillating in the cross-flow direction (Khalak \& Williamson 1999; Carberry et al. 2005), $\bar{C}_{x}$ tends to globally increase with the vibration amplitude. The deviation from the stationary body case value may become very large in the second vibration region, and cause 


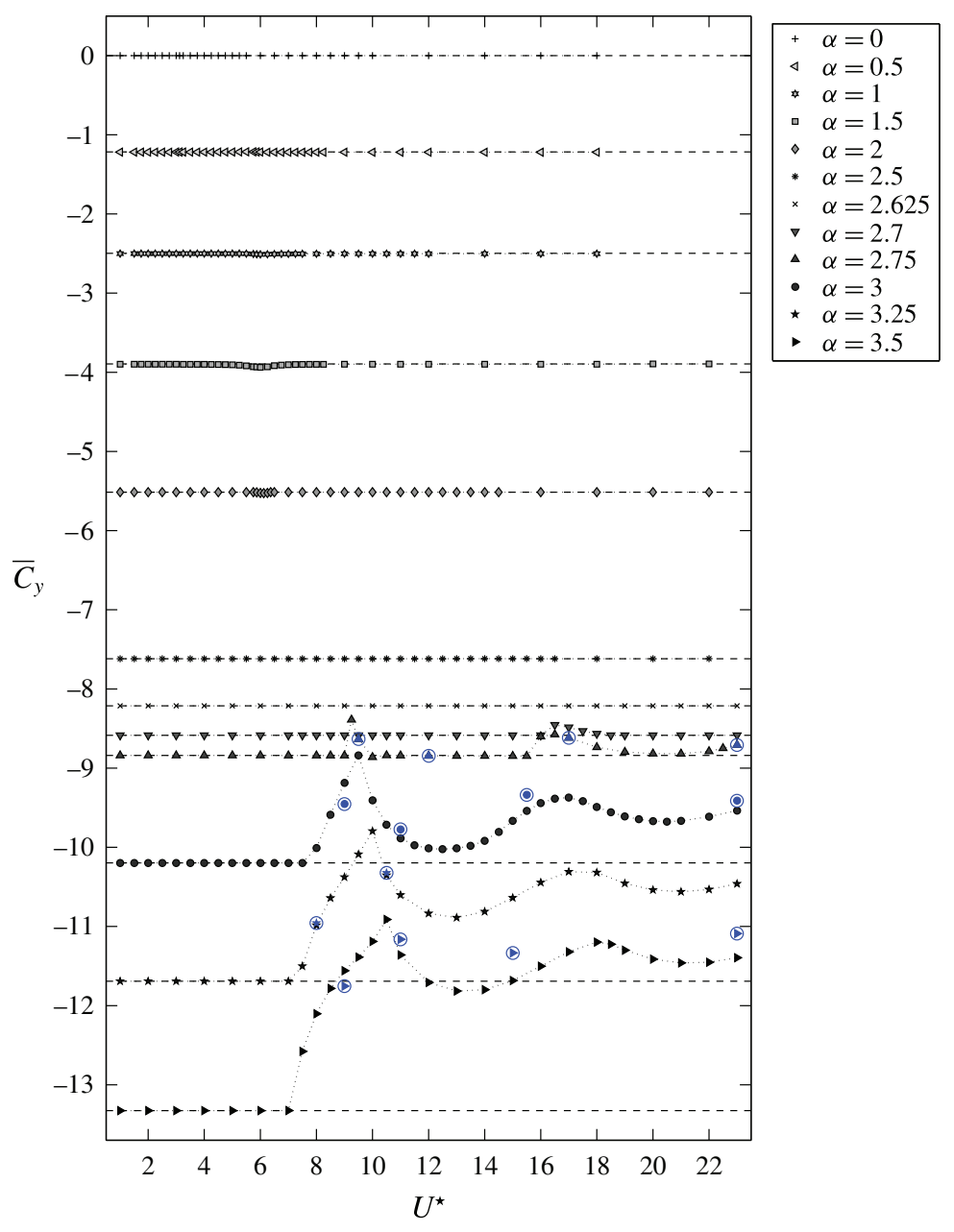

Figure 16. (Colour online) Same as figure 15 for the cross-flow force coefficient.

a substantial shift of the cylinder mean position $(\S 3.1)$. For a given rotation rate and comparable vibration amplitudes, some dispersion may appear in the values of $\bar{C}_{x}$, for instance, around $1.5 D$ for $\alpha=3.5$. Such dispersion is expected since the amplification of $\bar{C}_{x}$ is also connected to the vibration frequency, as reported in prior works (e.g. Carberry et al. 2005). In the cross-flow direction, small negative deviations of $\bar{C}_{y}$ from the stationary body case value can be noted within the first vibration region while the second vibration region is globally characterized by positive deviations of much larger magnitudes (figure 16).

The fluctuating parts of the fluid forces also exhibit considerable variations across the parameter space as illustrated in figure 18, which represents, for each rotation rate, the RMS values of the force coefficient fluctuations as functions of the reduced velocity. These RMS values, equal to zero beyond $\alpha=1.8$ in the absence of body oscillation due to the stationary nature of the flow, may reach high levels within the second vibration region $(\alpha \geqslant 2.7)$. A separate monitoring of the pressure and viscous components of the force fluctuation in the direction of motion (i.e. the in-line direction) shows that the RMS value of the pressure contribution follows the behaviour 

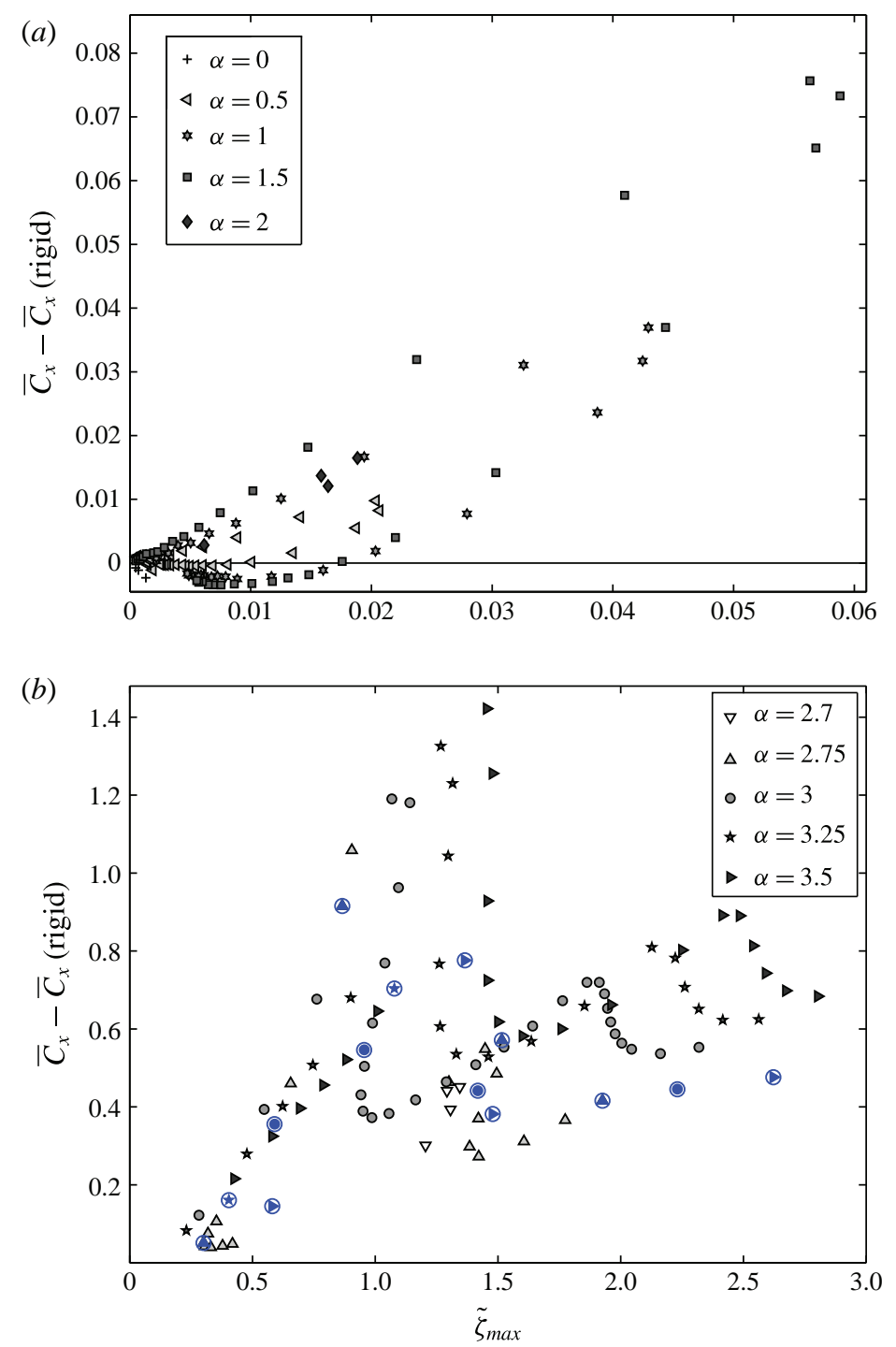

FIgURE 17. (Colour online) Deviation of the time-averaged in-line force coefficient from the value in the rigidly mounted cylinder case as a function of the maximum amplitude of vibration, for $(a) \alpha \leqslant 2$ and $(b) \alpha \geqslant 2.7$. In $(b)$, the three-dimensional simulation results are denoted by circled blue symbols.

of the total force while the viscous part presents generally a much lower RMS value and a smoother evolution as a function of $U^{\star}$. Such trends were previously observed for a rotating cylinder free to vibrate in the cross-flow direction (Bourguet \& Lo Jacono 2014).

The three-dimensional simulation results are indicated by circled blue symbols in figures 15-18. Beyond the critical rotation rate associated with the flow threedimensional transition $(\alpha \geqslant 3$ in the results presented), the time-averaged value of the in-line force coefficient and the RMS values of both force coefficient fluctuations are found to be globally lower than those predicted under two-dimensional flow 

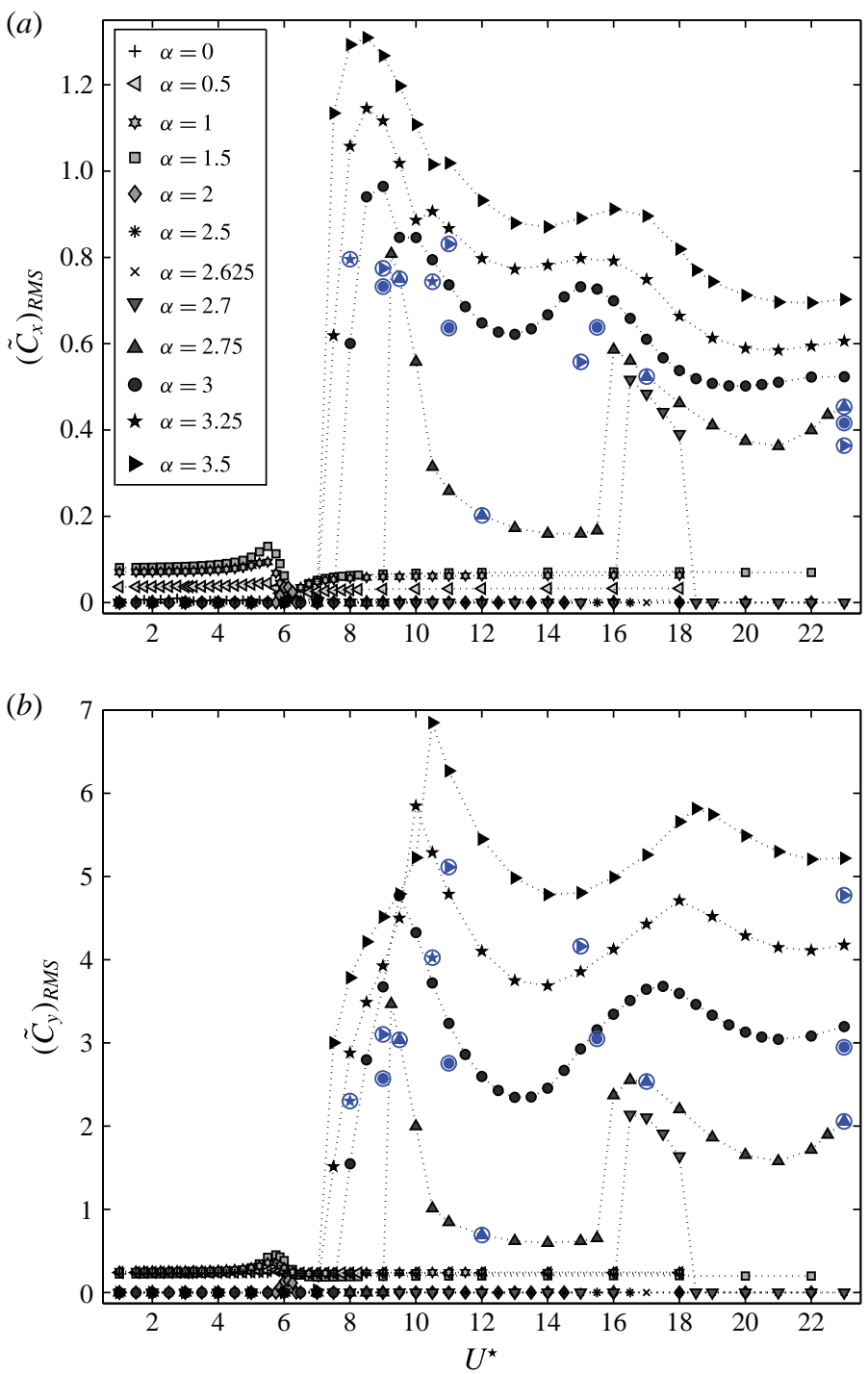

FIGURE 18. (Colour online) RMS $(a)$ in-line and $(b)$ cross-flow force coefficients as functions of the reduced velocity. The three-dimensional simulation results are denoted by circled blue symbols.

assumption. Nonetheless, the main features reported above concerning the fluid forcing, and especially the amplifications noted within the second vibration region, are confirmed by these additional three-dimensional results.

The fluid forces are periodic in all studied cases, similarly to the structural responses. The frequency content of the fluid force acting on the cylinder in the direction of motion is quantified in figure 19, where the PSD of $C_{x}$ time series is plotted as a function of the reduced velocity, for each rotation rate. The PSD is normalized by the magnitude of the largest peak. The fundamental frequency of the wake is denoted by a blue square. It can be observed that the fundamental frequency of the fluid forcing coincides with the fundamental frequency of the flow unsteadiness 


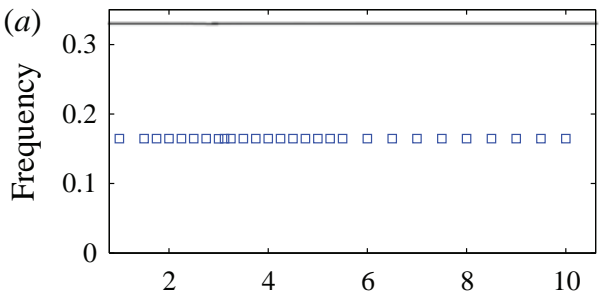

(b)
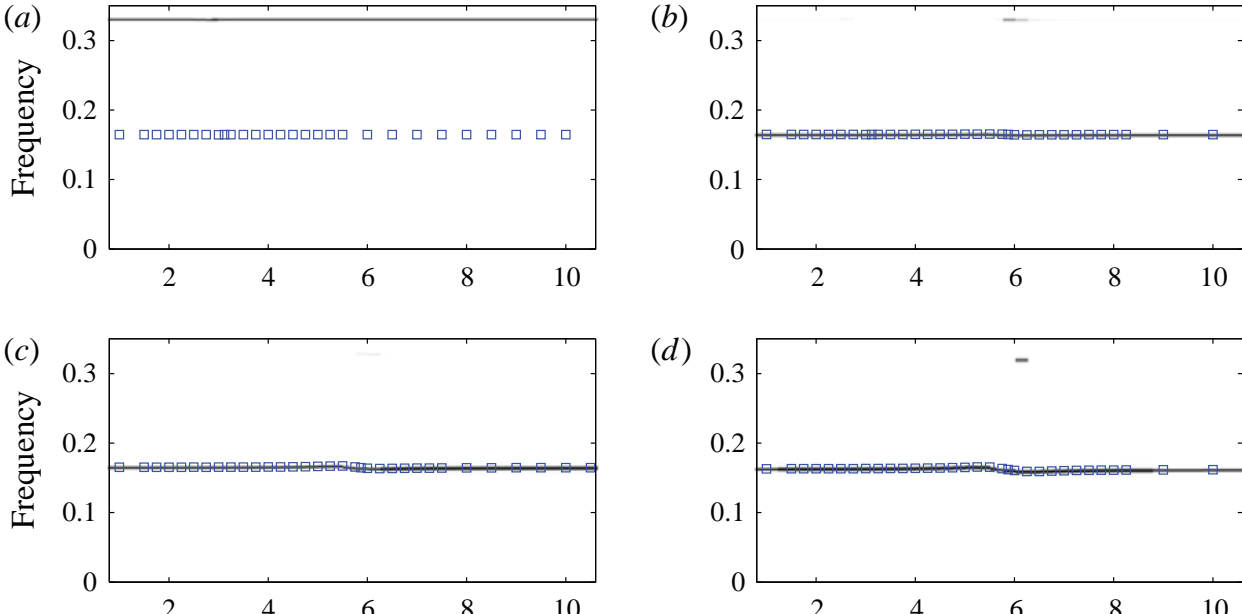

(d)
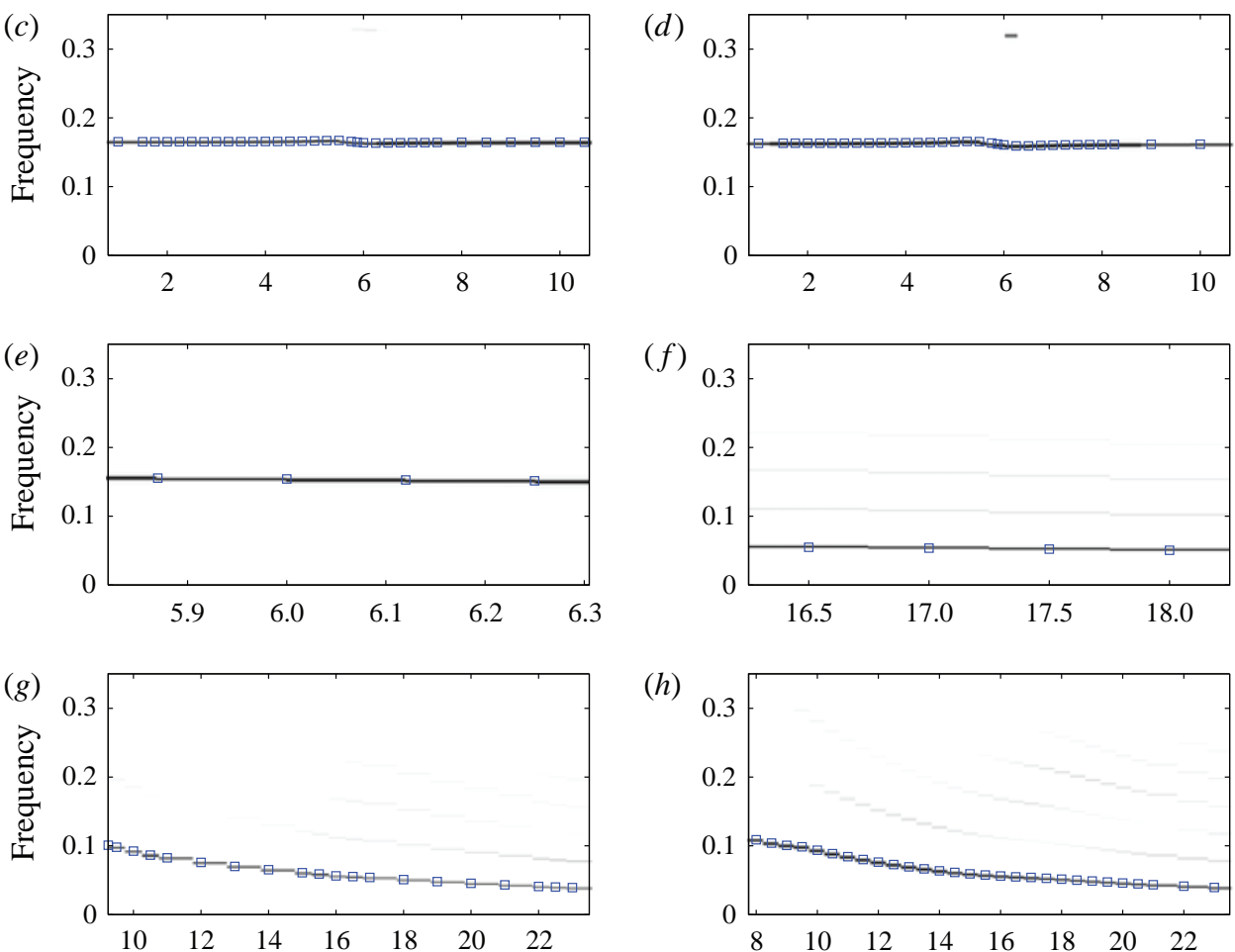

(h)
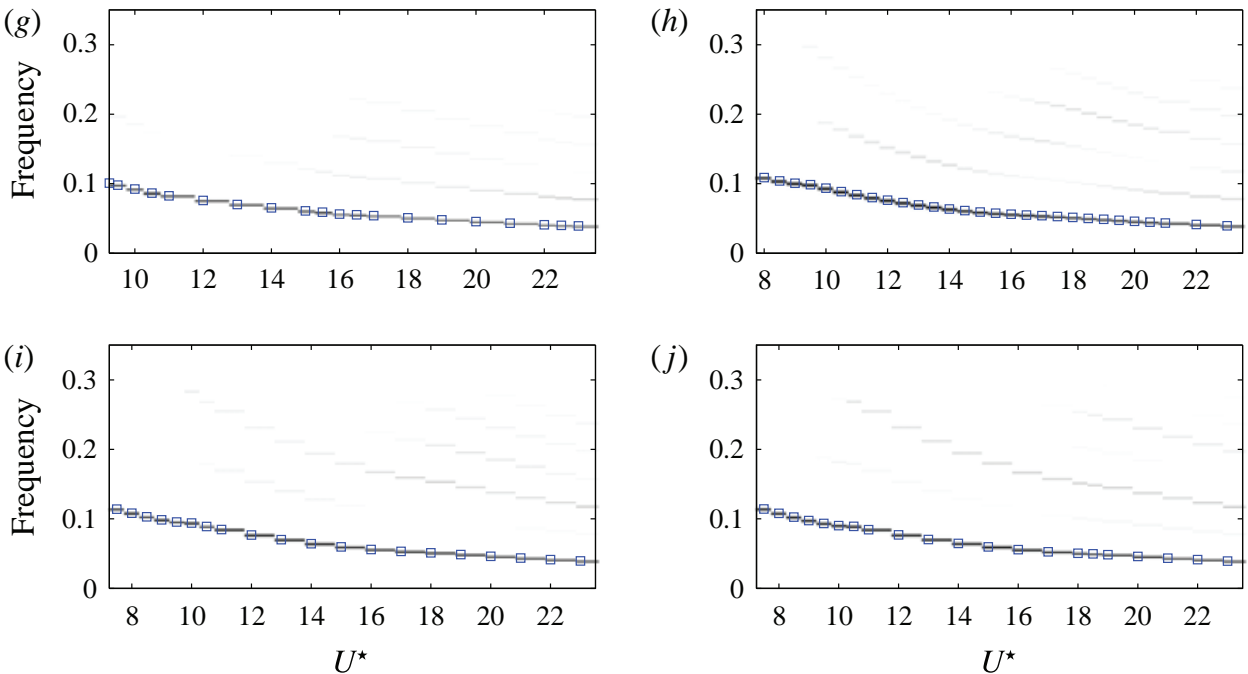

FIGURE 19. (Colour online) PSD of the in-line force coefficient as a function of the reduced velocity for $(a) \alpha=0,(b) \alpha=0.5,(c) \alpha=1,(d) \alpha=1.5,(e) \alpha=2,(f) \alpha=2.7$, (g) $\alpha=2.75,(h) \alpha=3$, (i) $\alpha=3.25$, (j) $\alpha=3.5$. For each reduced velocity, the PSD is normalized by the magnitude of the largest peak. The colour levels range from 0 (white) to 1 (black). The fundamental frequency of the wake is indicated by a blue square. 
or twice this frequency. It is recalled that the condition of wake-body synchronization is established in all studied cases $(\$ 4.1)$. Hence, the fluid forcing and the cylinder response are also synchronized. More preciselly, the fundamental frequencies of $C_{x}$ and $\zeta$ are the same. The global switch in the force-to-wake fundamental frequency ratio, from 2 to 1 between $\alpha=0$ and $\alpha>0$ cases, which is associated with the asymmetry introduced by the imposed rotation and which was previously reported for the structural response ( $\$ 3.2$ and 4.1), is clearly visible in this figure. Such frequency switch of the in-line force was also mentioned in prior studies concerning rigidly mounted rotating cylinders (e.g. Mittal \& Kumar 2003).

In previous work focusing on the free cross-flow vibrations of a rotating cylinder (Bourguet \& Lo Jacono 2014), fluid forcing was found to exhibit large higher harmonic components. This phenomenon was connected to the variable inclination of the flow velocity relative to the body frame due to the transverse displacement. Such mechanism does not apply in the present configuration where the direction of motion is aligned with the current, and it appears that the higher harmonic contributions remain generally small in the force spectrum. Some substantial second harmonic contributions may however be noted in a very narrow range of reduced velocities, around $U^{\star}=6$, within the first vibration region (e.g. for $\alpha=1.5$ ). This feature can be related to the force-displacement phasing as shown in the next subsection. In all the cases, the cross-flow force is dominated by a single component peaking at the fundamental wake frequency. The three-dimensional simulation results corroborate the above observations concerning the spectral content of the fluid forces.

\subsection{Force-displacement phasing and effective added mass}

The mechanisms of phasing between the fluid forcing and the free in-line oscillation of the rotating cylinder are analysed in the following. Due to their synchronization and the coincidence of their fundamental frequencies, the periodic in-line displacement and force coefficient may be expanded as follows:

$$
\zeta=\sum_{n=0}^{\infty} \zeta_{n} \sin \left(2 \pi n f_{x} t+\phi_{n}\right) \quad \text { and } \quad C_{x}=\sum_{n=0}^{\infty} C_{x n} \sin \left(2 \pi n f_{x} t+\phi_{x n}\right)
$$

where $\zeta_{n}$ and $C_{x n}$ designate the spectral amplitudes while $\phi_{n}$ and $\phi_{x n}$ denote the phase lags. Here, particular attention is paid to the phasing between the dominant vibration component and the associated force component. Since the principal vibration component may occur at $f=f_{x}$ or $f=2 f_{x}$, the generic index $n_{x}$ is used to designate the dominant vibration component of the cylinder $\left(f=n_{x} f_{x}\right)$. Without loss of generality, $\phi_{n_{x}}$ is selected equal to $0^{\circ}$ so that the phase difference between the force component associated with the main vibration component and this vibration component is equal to $\phi_{x n_{x}}$. If $\zeta$ and $C_{x}$ verify the dynamics equation (2.1) in which the structural damping is set to zero $(\xi=0)$, then (for $n_{x} f_{x} \neq f_{n}$ ):

$$
\phi_{x n_{x}}=0^{\circ} \text { or } 180^{\circ},
$$

and

$$
4 \pi^{2} f_{n}^{2}\left(1-f^{\star 2}\right) \zeta_{n_{x}}=\frac{C_{x n_{x}}}{2 m} \cos \left(\phi_{x n_{x}}\right),
$$

where, as previously defined, $f^{\star}=f / f_{n}=n_{x} f_{x} / f_{n}$. The two possible phasing states of the system are thus: (i) the force and displacement are in phase $\left(\phi_{x n_{x}}=0^{\circ}\right)$ and the 
vibration frequency is lower than the natural frequency in vacuum $\left(f^{\star}<1\right)$; (ii) the force and displacement are in antiphase $\left(\phi_{x n_{x}}=180^{\circ}\right)$ and the vibration frequency is larger than the natural frequency in vacuum $\left(f^{\star}>1\right)$. A phase jump occurs when the vibration frequency passes through the value of the natural frequency. For $f^{\star}=1$, the force component associated with the main displacement component $\left(C_{x n_{x}}\right)$ vanishes since $C_{x n_{x}} \cos \left(\phi_{x n_{x}}\right)=C_{x n_{x}} \sin \left(\phi_{x n_{x}}\right)=0$.

Previous works concerning non-rotating (Khalak \& Williamson 1999; Leontini et al. 2006; Dahl et al. 2010) and rotating (Bourguet \& Lo Jacono 2014; Zhao et al. 2014a) cylinders, oscillating either in the cross-flow direction or in both directions, have emphasized the possible existence of the two above phasing states. In the present case of free in-line vibrations, the cylinder vibration frequency was found to cross the oscillator natural frequency as $U^{\star}$ increases, for each rotation rate $\alpha \leqslant 1.5$ (figure $5 b$ ). As a result, both phasing states $\left(\phi_{x n_{x}}=0^{\circ}\right.$ and $\left.\phi_{x n_{x}}=180^{\circ}\right)$ may be encountered within the first vibration region. In contrast, the vibration frequency remains lower than the natural frequency in the second vibration region (figure 6b), which indicates that no phase jump occurs in this region and that the force and displacement are in phase, regardless the value of $U^{\star}$. This behaviour is also verified on the basis of the three-dimensional simulation results. The above observations are confirmed in figure 20, where the phase difference $\phi_{x n_{x}}$ is plotted as a function of the reduced velocity for each rotation rate (blue squares, left axis). An analogous disappearance of the force-displacement phase jump was previously reported beyond $\alpha=2$ for a rotating cylinder freely oscillating in the cross-flow direction (Bourguet \& Lo Jacono 2014). The phasing of the pressure and viscous parts of the in-line force can be monitored separately. The phase difference between the $n_{x}$ th harmonics of the pressure part of $C_{x}$ and the displacement is generally close to $\phi_{x n_{x}}$, with typical deviations in the range $0^{\circ}$ to $-30^{\circ}$. The viscous part exhibits larger deviations from $\phi_{x n_{x}}$. However, it is recalled that its contribution to $C_{x}$ fluctuation remains usually much smaller than the pressure part contribution.

On the basis of the above analysis, low magnitudes of the fundamental components of $C_{x}\left(C_{x 1}\right)$ are expected near the phase difference jumps identified within the first vibration region in figure $20\left(n_{x}=1\right.$ for all the phase difference jumps in this figure). Close to the jumps, the relative contributions of the higher harmonic components may thus become significant in the force spectrum, for instance, near $U^{\star}=6$, for $\alpha=0.5$ and $\alpha=1.5$ (figure 19).

The vibration frequency and force-displacement phasing are closely connected to the effective added mass coefficient, which can be defined as the ratio between the effective added mass induced by the fluid force in phase with the cylinder acceleration $\left(m_{a}\right)$ and the mass of the displaced fluid $\left(m_{f}\right)$ :

$$
C_{m}=\frac{m_{a}}{m_{f}}=-\frac{2}{\pi} \frac{\overline{C_{x} \ddot{\zeta}}}{\overline{\check{\zeta}^{2}}} \quad \text { with } m_{a}=-\frac{\overline{\left\langle F_{x}\right\rangle a}}{\overline{a^{2}}} \text { and } m_{f}=\rho_{f} \frac{\pi D^{2}}{4} \text {, }
$$

where $a$ denotes the dimensional acceleration $\left(a=\ddot{\zeta} U^{2} / D\right)$.

An effective added mass coefficient, involving the dominant component of the response only ( $n_{x}$ th harmonic), may be defined by removing all the other harmonic contributions in (5.4):

$$
C_{m}^{n_{x}}=\frac{C_{x n_{x}} \cos \left(\phi_{x n_{x}}\right)}{2 \pi^{3} f^{2} \zeta_{n_{x}}} .
$$



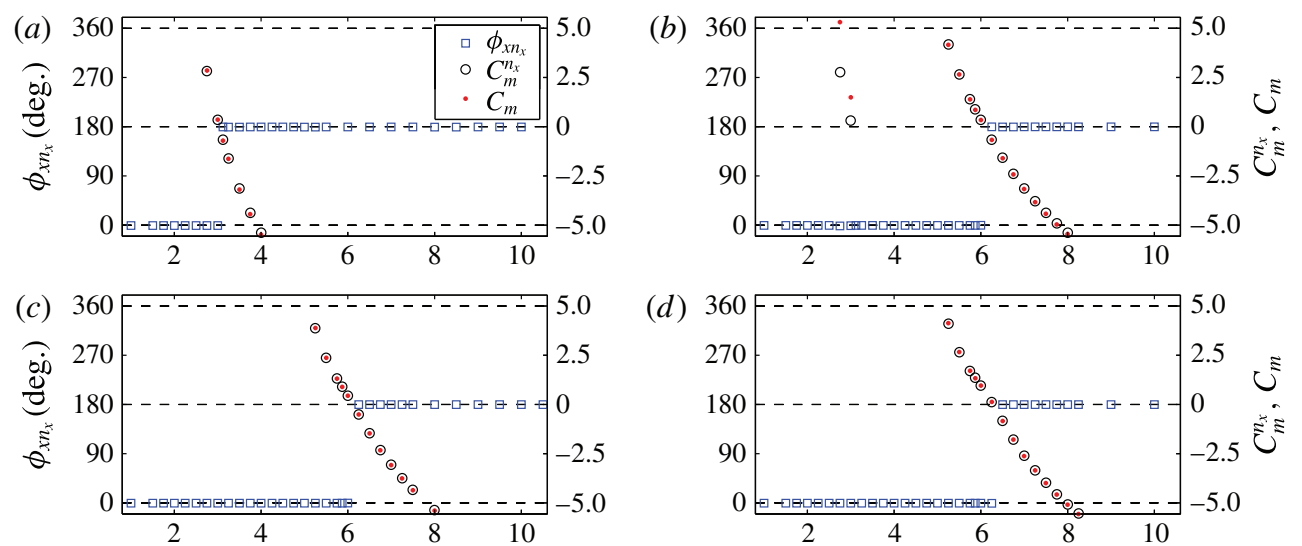

(d)
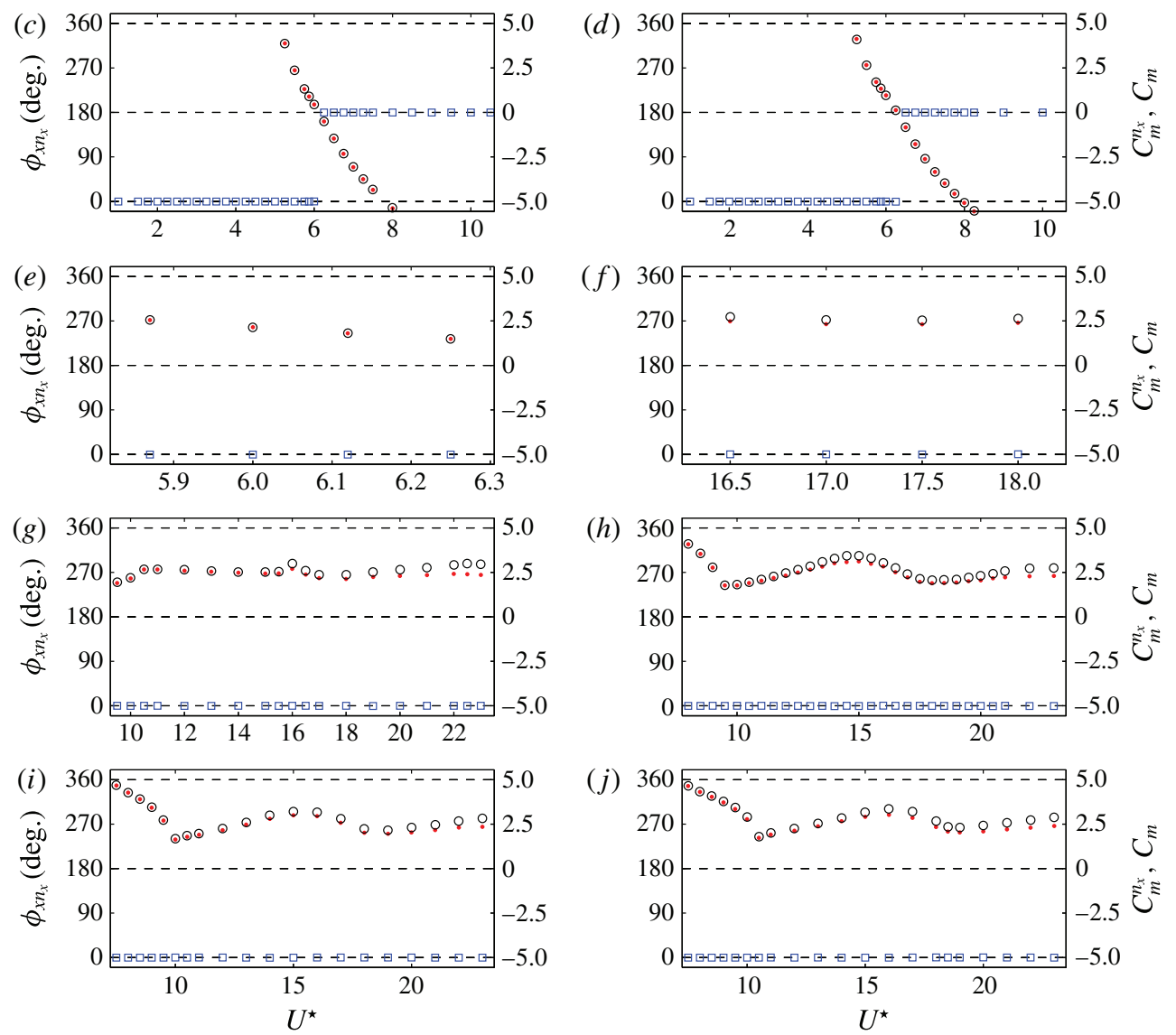

FIGURE 20. (Colour online) Phase difference between the in-line force coefficient and the cylinder displacement (left axis) and effective added mass coefficients (right axis), as functions of the reduced velocity for $(a) \alpha=0,(b) \alpha=0.5,(c) \alpha=1,(d) \alpha=1.5$, (e) $\alpha=2,(f) \alpha=2.7,(g) \alpha=2.75,(h) \alpha=3$, (i) $\alpha=3.25,(j) \alpha=3.5$. The effective added mass coefficient associated with the dominant harmonic of the response $\left(C_{m}^{n_{x}}\right.$ defined in (5.5)) and the effective added mass coefficient based on the total response $\left(C_{m}\right.$ defined in (5.4)) are denoted by a black circle and a red dot, respectively.

For sinusoidal oscillations, $C_{m}^{n_{x}}$ is equal to $C_{m}$. The coefficient $C_{m}^{n_{x}}$ relates to the vibration frequency as follows:

$$
f^{\star}=\sqrt{\frac{m}{m+\frac{\pi}{4} C_{m}^{n_{x}}}} .
$$


For each rotation rate, $C_{m}^{n_{x}}$ is plotted as a function of the reduced velocity in figure 20 (black circles, right axis) and the values of $C_{m}$ are also presented, for comparison purpose (red dots, right axis). The three-dimensional simulation results (not reported in this figure) confirm the trends described in the following. Due to the strong sinusoidal nature of the structural responses, $C_{m}$ remains generally close to $C_{m}^{n_{x}}$. Some differences may be noted for $\alpha=0.5$ near $U^{\star}=3$, where two vibration components of comparable magnitudes emerge in the response spectrum. As previously observed for a rotating cylinder oscillating in the cross-flow direction (Bourguet \& Lo Jacono 2014), the effective added mass coefficient substantially departs from the potential flow value of 1 . The global increase of the effective added mass coefficient with $\alpha$ identified in this prior work is not observed here. The decreasing trend and the sign change appearing within the first vibration region, for each $\alpha \leqslant 1.5$, when $U^{\star}$ increases, is in agreement with the increasing trend of the vibration frequency, which crosses the oscillator natural frequency (figure $5 b$ ). The positive value of the effective added mass for $\alpha \geqslant 2$ is associated with a vibration frequency that remains lower than the natural frequency $\left(f^{\star}<1\right)$ and thus with the absence of jump of the force-displacement phase difference in the second vibration region.

\section{Conclusions}

The flow-induced vibrations of a circular cylinder, free to oscillate in the in-line direction and subjected to a forced rotation, have been investigated over a wide range of rotation rates and reduced velocities, at a Reynolds number equal to 100 . A joint analysis of the structural responses, wake patterns and fluid forces was presented on the basis of two- and three-dimensional simulation results. Free in-line oscillations occur below the rotation rate associated with vortex shedding suppression in the rigidly mounted cylinder case $(\alpha \approx 1.8)$ but also beyond this critical value of $\alpha$. Two regions of vibrations have been identified in the $\left(\alpha, U^{\star}\right)$ parameter space, for rotation rates lower than 2 and larger than 2.7 approximately, each one characterized by a specific regime of response. Between the vibration regions, the steady wake is composed of two elongated layers of spanwise vorticity of opposite signs $\left(\mathrm{D}^{+}\right.$ pattern). The principal features of the two vibration regions are summarized hereafter.

First vibration region: VIV-like responses. From $\alpha=0$ up to a rotation rate close to 2 , the cylinder is found to exhibit low-amplitude vibrations. For each rotation rate in this zone, the oscillation amplitude follows a bell-shaped evolution as a function of the reduced velocity. The maximum vibration amplitudes are observed around $U^{\star}=6$. They reach $0.06 D$ for $\alpha=1.5$, which represents a considerable amplification compared to the non-rotating body case where a maximum vibration amplitude of $1.5 \times 10^{-3} \mathrm{D}$ is noted at $R e=100$. Due to their trend as functions of $U^{\star}$ and to the fact that they develop under a condition of wake-body synchronization or lock-in, the responses of the rotating cylinder in this region are comparable to the VIV previously described for non-rotating cylinders.

The typical wake pattern in this region consists of two counter-rotating vortices shed per cycle. The alteration of the wake anti-symmetry induced by the rotation is generally accompanied by a switch of the in-line fluid force and body oscillation frequencies, which are equal to the fundamental frequency of the flow unsteadiness, versus twice this frequency for $\alpha=0$. For a given rotation rate, both forcedisplacement phasing states (i.e. in-line force and displacement in phase or in antiphase) may be encountered in this area of the parameter space, depending on the value of $U^{\star}$. The phase difference jumps associated with transitions between these 
two states, when the vibration frequency crosses the oscillator natural frequency and the effective added mass changes sign, may thus occur in the first vibration region.

Second vibration region: galloping-like responses. The lower limit of the second vibration region lies close to $\alpha=2.7$. The vibrations appear around $U^{\star}=8$. Contrary to the bell-shaped evolutions noted in the first vibration region, the amplitude of the typical response observed in this second region tends to increase unboundedly with $U^{\star}$. It may become very large, e.g. higher than $2.5 D$ for $\left(\alpha, U^{\star}\right)=(3.5,23)$. Therefore, the structural oscillations developing in this area of the parameter space resemble the galloping responses reported for non-axisymmetric bodies. These vibrations, however, cannot be predicted through quasi-steady analysis. Galloping-like oscillations were previously observed for a rotating circular cylinder free to move in both the in-line and cross-flow directions but not when the body was restrained to a single degree-of-freedom in the cross-flow direction. For a given reduced velocity, the oscillation amplitude is found to increase with $\alpha$.

As in the first vibration region, body oscillation and flow unsteadiness remain synchronized across the entire response area. Such synchronization is not generally expected for galloping oscillations. Here, the vibration frequency is shown to always coincide with the fundamental frequency of the wake. The number of vortices shed per cycle tends to globally increase with the vibration amplitude and with the reduction of its frequency. Novel asymmetric multi-vortex patterns, combining a pair and a triplet or a quartet of vortices per cycle, develop in this region. The flow past the oscillating cylinder is found to undergo three-dimensional transition for a critical rotation rate located between 2.75 and 3, i.e. lower than for a rigidly mounted cylinder $(\alpha \approx 3.7)$. The flow three-dimensional transition is characterized by a regular spanwise undulation particularly pronounced around the body and in the near wake, and which tends to vanish further downstream. The spanwise wavelength of the three-dimensional pattern ranges between $1 D$ and $1.7 D$ approximately. It should be mentioned that the three-dimensional nature of the flow has only a limited influence on the system behaviour since the three-dimensional simulation results beyond the critical rotation rate are close to those obtained under two-dimensional flow assumption.

The fluid forces, which mainly peak at the vibration/wake frequency, are considerably altered in this zone of large-amplitude oscillations, compared to the non-vibrating body case. In particular, the time-averaged in-line force is substantially amplified and this amplification is associated with a shift of the cylinder mean position. Within the second vibration region, the force and displacement are shown to be in phase, i.e. no phase difference jump occurs. This phenomenon is related to a vibration frequency that remains lower than the oscillator natural frequency, and a positive value of the effective added mass.

\section{Acknowledgements}

This work was performed using HPC resources from CALMIP (grants 2014-P1248 and 2015-P1248) and GENCI (grants x20142a7184 and x20152a7184).

\section{Appendix A. Quasi-steady analysis}

Additional results are presented in this appendix to clarify the possibility of predicting through quasi-steady approach, the large-amplitude responses encountered in the second vibration region identified in §3.2. A series of two-dimensional 


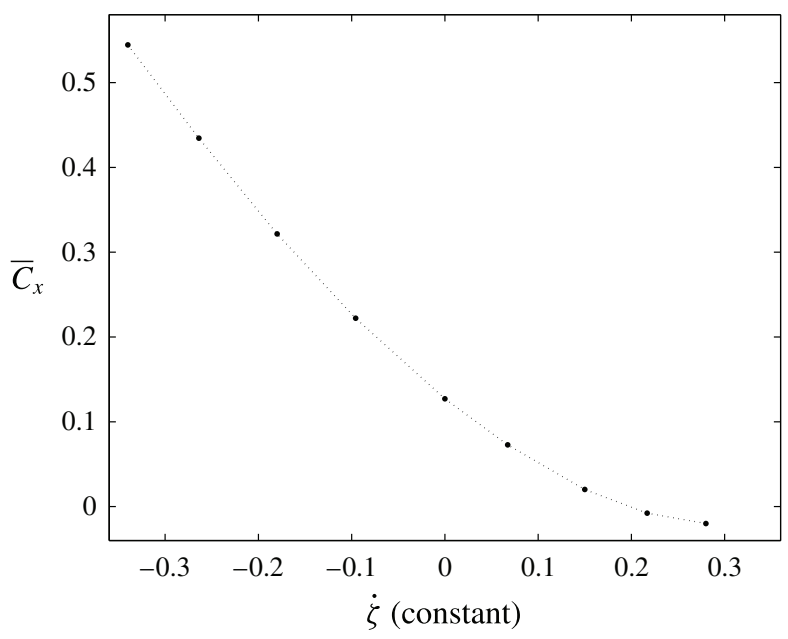

FIGURE 21. Time-averaged in-line force coefficient as a function of the cylinder velocity for a cylinder moving at constant velocity $(\alpha=3)$.

simulations of the flow past a rotating cylinder subjected to a constant velocity $\dot{\zeta}$ have been performed over a range of body velocities around $\dot{\zeta}=0$. The rotation rate was set to a value of 3, for which the flexibly mounted cylinder is subjected to galloping-like oscillations typical of the second vibration region.

The time-averaged in-line force coefficient obtained by these simulations is plotted in figure 21 as a function of the body velocity. The flow is steady in all these additional simulations and $\bar{C}_{x}=C_{x}$. The in-line force decreases with the cylinder velocity in the range investigated. These results obtained for a cylinder moving at constant velocity thus suggest that the fluid force would tend to oppose any variation of $\dot{\zeta}$ around $\dot{\zeta}=0$. Therefore, the cylinder responses identified when it is free to oscillate are not expected based on the above quasi-steady analysis.

\section{Appendix B. Complements on three-dimensional transition}

Instantaneous rotation rate and Reynolds number, based on the instantaneous flow velocity in the cylinder frame, can be defined respectively as follows:

$$
\alpha_{i}=\frac{\alpha}{1-\dot{\zeta}} \quad \text { and } \quad \operatorname{Re}_{i}=\operatorname{Re}(1-\dot{\zeta}) .
$$

The evolutions of $\alpha_{i}$ and $R e_{i}$ over one oscillation cycle are plotted in figure 22 for four selected points located in the second vibration region (three-dimensional simulation results). In this plot, the limit between two- and three-dimensional flow determined by Pralits et al. (2013) and Rao et al. (2013a) in the rigidly mounted cylinder configuration is indicated by a dashed line. The vibrating body crosses this limit in all selected cases. However, the flow which is found to be three-dimensional in the last three cases $(\alpha \geqslant 3)$, remains two-dimensional in the first one $(\alpha=2.75)$. In addition, it can be noted that the excursion beyond this limit is longer for $\left(\alpha, U^{\star}\right)=(2.75,9.5)$ than for $\left(\alpha, U^{\star}\right)=(3.25,8)$ whereas the flow is two-dimensional in the former case and three-dimensional in the latter case. As a consequence, it appears that the twoor three-dimensional nature of the flow past the vibrating rotating cylinder cannot be anticipated from the results obtained in the rigidly mounted body configuration. 


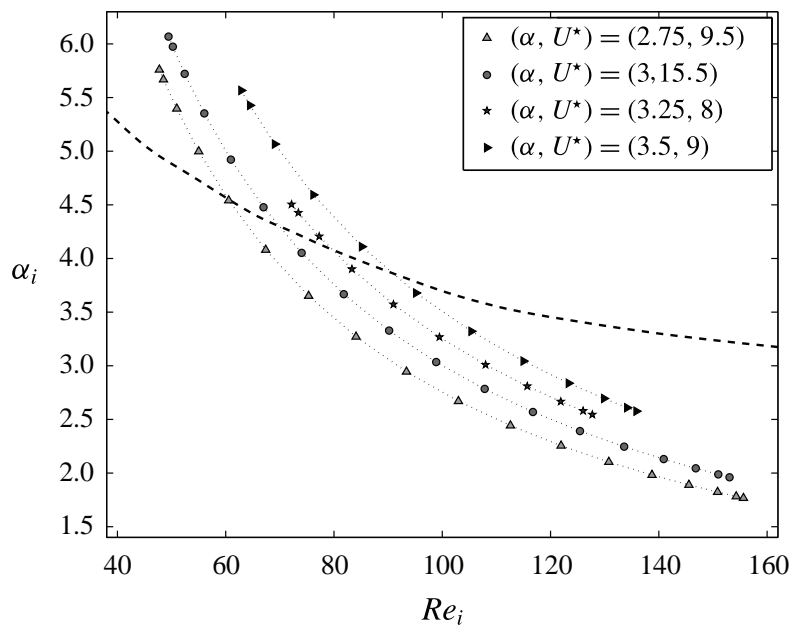

FIGURE 22. Instantaneous rotation rate and Reynolds number based on flow velocity in the cylinder frame, over one oscillation cycle, for selected points in the second vibration region. The onset of flow three-dimensionality reported by Pralits et al. (2013) and Rao et al. (2013a) for a rigidly mounted rotating cylinder is indicated by a dashed line.

\section{REFERENCES}

Abdel-Rohman, M. 1992 Galloping of tall prismatic structures: a two-dimensional analysis. J. Sound Vib. 153, 97-111.

Badr, H. M., Coutanceau, M., Dennis, S. C. R. \& Ménard, C. 1990 Unsteady flow past a rotating circular cylinder at Reynolds numbers $10^{3}$ and $10^{4}$. J. Fluid Mech. 220, 459-484.

Bearman, P. W. 1984 Vortex shedding from oscillating bluff bodies. Annu. Rev. Fluid Mech. 16, $195-222$.

Bearman, P. W. 2011 Circular cylinder wakes and vortex-induced vibrations. J. Fluids Struct. 27, 648-658.

Bearman, P. W., Gartshore, I. S., Maull, D. J. \& Parkinson, G. V. 1987 Experiments on flow-induced vibration of a square-section cylinder. J. Fluids Struct. 1, 19-34.

Bishop, R. E. D. \& HASSAN, A. Y. 1964 The lift and drag forces on a circular cylinder oscillating in a flowing fluid. Proc. R. Soc. Lond. A 277, 51-75.

Blevins, R. D. 1990 Flow-induced Vibration. Van Nostrand Reinhold.

Bourguet, R., Karniadakis, G. E. \& Triantafyllou, M. S. 2011 Vortex-induced vibrations of a long flexible cylinder in shear flow. J. Fluid Mech. 677, 342-382.

Bourguet, R. \& LO JACONO, D. 2014 Flow-induced vibrations of a rotating cylinder. J. Fluid Mech. 740, 342-380.

BRiKA, D. \& LANEVILle, A. 1993 Vortex-induced vibrations of a long flexible circular cylinder. J. Fluid Mech. 250, 481-508.

Cagney, N. \& Balabani, S. 2013 Wake modes of a cylinder undergoing free streamwise vortexinduced vibrations. J. Fluids Struct. 38, 127-145.

Carberry, J., Sheridan, J. \& Rockwell, D. 2001 Forces and wake modes of an oscillating cylinder. J. Fluids Struct. 15, 523-532.

CARberry, J., Sheridan, J. \& RockWell, D. 2005 Controlled oscillations of a cylinder: forces and wake modes. J. Fluid Mech. 538, 31-69.

Cetiner, O. \& Rockwell, D. 2001 Streamwise oscillations of a cylinder in a steady current. Part 1. Locked-on states of vortex formation and loading. J. Fluid Mech. 427, 1-28.

Chew, Y. T., Cheng, M. \& LuO, S. C. 1995 A numerical study of flow past a rotating circular cylinder using a hybrid vortex scheme. J. Fluid Mech. 299, 35-71. 
Corless, R. M. \& Parkinson, G. V. 1988 A model of the combined effects of vortex-induced oscillation and galloping. J. Fluids Struct. 2, 203-220.

Coutanceau, M. \& MÉnARD, C. 1985 Influence of rotation on the near-wake development behind an impulsively started circular cylinder. J. Fluid Mech. 158, 399-446.

Dahl, J. M., Hover, F. S., Triantafyllou, M. S. \& OAKley, O. H. 2010 Dual resonance in vortex-induced vibrations at subcritical and supercritical Reynolds numbers. J. Fluid Mech. 643, 395-424.

Den Hartog, J. P. 1932 Transmission line vibration due to sleet. Trans. Am. Inst. Electr. Engrs 51, 1074-1076.

El Akoury, R., Braza, M., Perrin, R., Harran, G. \& Hoarau, Y. 2008 The three-dimensional transition in the flow around a rotating cylinder. J. Fluid Mech. 607, 1-11.

Govardhan, R.\& Williamson, C. H. K. 2000 Modes of vortex formation and frequency response of a freely vibrating cylinder. J. Fluid Mech. 420, 85-130.

GRIFFIN, O. M. \& RAMBERG, S. E. 1976 Vortex shedding from a cylinder vibrating in line with an incident uniform flow. J. Fluid Mech. 75, 257-271.

Hover, F. S., Techet, A. H. \& Triantafyllou, M. S. 1998 Forces on oscillating uniform and tapered cylinders in crossflow. J. Fluid Mech. 363, 97-114.

JEON, D. \& GHARIB, M. 2001 On circular cylinders undergoing two-degree-of-freedom forced motions. J. Fluids Struct. 15, 533-541.

KAng, S., ChOI, H. \& LeE, S. 1999 Laminar flow past a rotating circular cylinder. Phys. Fluids 11, 3312 .

Karniadakis, G. E. \& Sherwin, S. 1999 Spectral/hp Element Methods for CFD, 1st edn. Oxford University Press.

Khalak, A. \& Williamson, C. H. K. 1999 Motions, forces and mode transitions in vortex-induced vibrations at low mass-damping. J. Fluids Struct. 13, 813-851.

King, R., Prosser, M. J. \& Johns, D. J. 1973 On vortex excitation of model piles in water. J. Sound Vib. 29, 169-188.

Klamo, J. T., Leonard, A. \& Roshko, A. 2006 The effects of damping on the amplitude and frequency response of a freely vibrating cylinder in cross-flow. J. Fluids Struct. 22, 845-856.

Konstantinidis, E. 2014 On the response and wake modes of a cylinder undergoing streamwise vortex-induced vibration. J. Fluids Struct. 45, 256-262.

Leontini, J. S., Lo JACOno, D. \& Thompson, M. C. 2011 A numerical study of an inline oscillating cylinder in a free stream. J. Fluid Mech. 688, 551-568.

Leontini, J. S., Lo Jacono, D. \& Thompson, M. C. 2013 Wake states and frequency selection of a streamwise oscillating cylinder. J. Fluid Mech. 730, 162-192.

Leontini, J. S., Thompson, M. C. \& Hourigan, K. 2006 The beginning of branching behaviour of vortex-induced vibration during two-dimensional flow. J. Fluids Struct. 22, 857-864.

Lucor, D. \& Triantafyllou, M. S. 2008 Parametric study of a two degree-of-freedom cylinder subject to vortex-induced vibrations. J. Fluids Struct. 24, 1284-1293.

Mannini, C., Marra, A. M. \& BARtoli, G. 2014 VIV-galloping instability of rectangular cylinders: review and new experiments. J. Wind Engng Ind. Aerodyn. 132, 109-124.

Meena, J., Sidarth, G. S., Khan, M. H. \& Mittal, S. 2011 Three-dimensional instabilities in flow past a spinning and translating cylinder. In IUTAM Symposium on Bluff Body Flows, IIT-Kanpur, India (ed. S. Mittal \& G. Biswas), pp. 75-78.

Mittal, S. 2004 A finite element study of incompressible flows past oscillating cylinders and aerofoils. J. Appl. Mech. 71, 89-95.

Mittal, S. \& Kumar, B. 2003 Flow past a rotating cylinder. J. Fluid Mech. 476, 303-334.

Mittal, S. \& TezduYAR, T. E. 1992 A finite element study of incompressible flows past oscillating cylinders and aerofoils. Intl J. Numer. Meth. Fluids 15, 1073-1118.

Mukhopadhyay, V. \& Dugundji, J. 1976 Wind excited vibration of a square section cantilever beam in smooth flow. J. Sound Vib. 45, 329-339.

NAKAmURA, Y. \& Tomonari, Y. 1977 Galloping of rectangular prisms in a smooth and a turbulent flow. J. Sound Vib. 52, 233-241.

NAUdAscher, E. 1987 Flow-induced streamwise vibrations of structures. J. Fluids Struct. 1, 265-298. 
Naudascher, E. \& Rockwell, D. 1994 Flow-induced Vibrations: An Engineering Guide. Dover. NAVRose \& MitTal, S. 2013 Free vibrations of a cylinder: 3-D computations at $R e=1000$. J. Fluids Struct. 41, 109-118.

Nemes, A., Zhao, J., Lo Jacono, D. \& Sheridan, J. 2012 The interaction between flow-induced vibration mechanisms of a square cylinder with varying angles of attack. J. Fluid Mech. 710, $102-130$.

Obasaju, E. D., Ermshaus, R. \& Naudascher, E. 1990 Vortex-induced streamwise oscillations of a square-section cylinder in a uniform stream. J. Fluid Mech. 213, 171-189.

Okajima, A., Kosugi, T. \& NAKAmura, A. 2002 Flow-induced in-line oscillation of a circular cylinder in a water tunnel. Trans. ASME: J. Press. Vessel Technol. 124, 89-96.

Okajima, A., Ohtsuyama, S., NAgAmori, T., NAKAno, T. \& Kiwata, T. 1999 In-line oscillation of structure with a circular or rectangular section. Trans. Japan. Soc. Mech. Engng 65, 2196-2203.

Ongoren, A. \& Rockwell, D. 1988 Flow structure from an oscillating cylinder. Part 2. Mode competition in the near wake. J. Fluid Mech. 191, 225-245.

Païdoussis, M. P., Price, S. J. \& De LAngre, E. 2010 Fluid-Structure Interactions: Cross-FlowInduced Instabilities. Cambridge University Press.

PARKInSON, G. V. \& SMITH, J. D. 1964 The square prism as an aeroelastic nonlinear oscillator. Q. J. Mech. Appl. Maths 17, 225-239.

Perdikaris, P. G., Kaiktsis, L. \& Triantafyllou, G. S. 2009 Chaos in a cylinder wake due to forcing at the Strouhal frequency. Phys. Fluids 21, 101705.

Pralits, J. O., Brandt, L. \& Giannetti, F. 2010 Instability and sensitivity of the flow around a rotating circular cylinder. J. Fluid Mech. 650, 513-536.

Pralits, J. O., Giannetti, F. \& BrandT, L. 2013 Three-dimensional instability of the flow around a rotating circular cylinder. J. Fluid Mech. 730, 5-18.

PRANDTL, L. 1925 Application of the 'Magnus effect' to the wind propulsion of ships. Naturwissenschaft 13, 93-108 (NACA Technical Memorandum, 367).

Prasanth, T. K. \& Mittal, S. 2008 Vortex-induced vibrations of a circular cylinder at low Reynolds numbers. J. Fluid Mech. 594, 463-491.

Radi, A., Thompson, M. C., Rao, A., Hourigan, K. \& Sheridan, J. 2013 Experimental evidence of new three-dimensional modes in the wake of a rotating cylinder. J. Fluid Mech. 734, $567-594$.

Rao, A., Leontini, J. S., Thompson, M. C. \& Hourigan, K. $2013 a$ Three-dimensionality in the wake of a rapidly rotating cylinder in uniform flow. J. Fluid Mech. 730, 379-391.

Rao, A., Leontini, J. S., Thompson, M. C. \& Hourigan, K. $2013 b$ Three-dimensionality in the wake of a rotating cylinder in a uniform flow. J. Fluid Mech. 717, 1-29.

Rao, A., Radi, A., Leontini, J. S., Thompson, M. C., Sheridan, J. \& Hourigan, K. 2015 A review of rotating cylinder wake transitions. J. Fluids Struct. 53, 2-14.

SARPKAYA, T. 2004 A critical review of the intrinsic nature of vortex-induced vibrations. J. Fluids Struct. 19, 389-447.

StAnsby, P. K. \& RAiney, R. C. T. 2001 On the orbital response of a rotating cylinder in a current. J. Fluid Mech. 439, 87-108.

Stojković, D., Breuer, M. \& Durst, F. 2002 Effect of high rotation rates on the laminar flow around a circular cylinder. Phys. Fluids 14, 3160.

Stojković, D., Schön, P., BReuer, M. \& Durst, F. 2003 On the new vortex shedding mode past a rotating circular cylinder. Phys. Fluids 15, 1257.

Tudball-Smith, D., Leontini, J. S., Sheridan, J. \& Lo Jacono, D. 2012 Streamwise forced oscillations of circular and square cylinders. Phys. Fluids 24, 111703.

Williamson, C. H. K. \& Govardhan, R. 2004 Vortex-induced vibrations. Annu. Rev. Fluid Mech. 36, 413-455.

Williamson, C. H. K. \& RoshKo, A. 1988 Vortex formation in the wake of an oscillating cylinder. J. Fluids Struct. 2, 355-381. 
Yogeswaran, V. \& MitTaL, S. 2011 Vortex-induced and galloping response of a rotating circular cylinder. In IUTAM Symposium on Bluff Body Flows, IIT-Kanpur, India (ed. S. Mittal \& G. Biswas), pp. 153-156.

Zhao, J., Leontini, J. S., Lo Jacono, D. \& Sheridan, J. $2014 a$ Fluid-structure interaction of a square cylinder at different angles of attack. J. Fluid Mech. 747, 688-721.

Zhao, M., Cheng, L. \& LU, L. $2014 b$ Vortex induced vibrations of a rotating circular cylinder at low Reynolds number. Phys. Fluids 26, 073602.

Zhao, M., Cheng, L. \& Zhou, T. 2013 Numerical simulation of vortex-induced vibration of a square cylinder at a low Reynolds number. Phys. Fluids 25, 023603. 